Review

\title{
Extension of the super-elements method to the analysis of oblique collision between two ships
}

\author{
Loïc Buldgen $^{\mathrm{a}, 1, *}$, Hervé Le Sourne ${ }^{\mathrm{b}}$, Nicolas Besnard ${ }^{\mathrm{c}}$, Philippe Rigo ${ }^{\mathrm{d}}$ \\ a University of Liège, ANAST, 1 Chemin des Chevreuils, 4000 Liège, Belgium \\ ${ }^{\mathrm{b}}$ ICAM Nantes, 35 Avenue du champ de Manœuvres, 44470 Carquefou, France \\ ${ }^{\mathrm{C}}$ PRINCIPIA, 1 rue de la Noé, 44321 NANTES Cedex 3, France \\ ${ }^{\mathrm{d}}$ University of Liège, ANAST, 1 Chemin des Chevreuils, 4000 Liège, Belgium
}

\section{A R T I C L E I $\mathrm{N}$ F $\mathrm{O}$}

Article history:

Received 29 July 2011

Received in revised form 18 July 2012

Accepted 7 August 2012

\section{Keywords:}

Oblique ship collision

Crashworthiness

Plastic method

Internal mechanics

\begin{abstract}
A B S T R A C T
The present article is concerned with the resistance of a ship during a collision with another ship. The paper is directly related to the so called super-elements method developed to estimate the capability of a ship to withstand to an impact. This approach consists of dividing the structure into several elements (the socalled super-elements), whose resistance to collision is evaluated individually. At the recent stage of development, the superelements method is only able to treat the case of perpendicular collision scenarios. The purpose of this paper is to go one step further, by establishing analytical formulations giving the resistance of various super-elements to an oblique impact.

As a first step, the paper gives a short description of the original super-elements method. After that, analytical calculations in oblique collision cases are performed for the different superelements involved in the procedure. Finally, the formulations are validated by comparison with results provided by classical nonlinear finite element method. As a conclusion, some perspectives on the future work are presented.
\end{abstract}

() 2012 Elsevier Ltd. All rights reserved.

\footnotetext{
* Corresponding author. University of Liège, Faculty of Applied Sciences, ANAST, 1 Chemin des Chevreuils, 4000 Liège, Belgium. Tel.: +32 (0) 436648 59, +32 (0) 478502188 (mobile); fax: +32 (0) 43669133.

E-mail addresses: L.Buldgen@ulg.ac.be (L. Buldgen), herve.lesourne@icam.fr (H. Le Sourne), nicolas.besnard@principia.fr (N. Besnard), ph.rigo@ulg.ac.be (P. Rigo).

${ }^{1}$ FRIA PhD Student
} 


\section{Introduction}

Amongst all the loads which have to be expected for the design of a ship, the collision with another vessel is one of the most important. This is especially the case for cargo vessels and tankers, which are devoted to the transport of oil, petrol or other toxic products, and may induce a severe pollution of oceans, such as during oil slicks for example. These environmental disasters have to be avoided, principally because of their consequences on marine biotopes, but also because they are economically and humanly expensive. Moreover, the reputation of the companies involved in these ecological degradations can be severely damaged.

To deal properly with ship collision, it is of course possible to use nonlinear finite element methods. Nevertheless, at the pre-design stage, such approaches are rather prohibitive because of the time required to model and simulate collisions involving large-size structures. This is especially true when a large number of scenarios have to be investigated. Therefore, simplified methods (empirical or analytical) have been developed to verify the resistance of ships submitted to impacts (see Pedersen [1] for a detailed review of the existing methods).

Minorsky [2] was the first to establish a simplified formulation of the resistance to collision. His formula was based on statistical data and was only valid for large ships. Since this pioneer work, some more refined analytical developments have been performed in order to assess the impact resistance of various structural elements of ships. These individual members may be classified in three main categories:

- the web girders, such as decks, stringers, transverse frame, transverse bulkheads, bottom floors, longitudinal girders,...

- the side panels, which are used to model the behavior of the outer and the inner shell plating (if any) in collision analysis.

- the intersection elements which are located at the junction between vertical and horizontal structural members.

In the literature, various authors have already developed theoretical models of all the previous components involved in naval architecture. For example, the crushing resistance of web girders was theoretically and experimentally studied by Wierzbicki and Culbertson-Driscoll [3], Wang and Ohtsubo [4], Simonsen [5] and Zhang [6]. Hong and Amdahl [7] summarized and compared all these various approaches.

The individual behavior of ship side panels has been investigated in details by Wang $[8,9]$ and Zhang [6]. Some references are also available to evaluate the resistance of metal plates after rupture, when they are submitted to tearing and cutting. For example, these phenomena have been studied by Wang [10], Zhang [11], Wierzbicki [12] and Zheng [13].

Finally, the crushing resistance of the intersection between vertical and structural members has been analyzed in details by Amdahl [17] and Zhang [6].

The previous brief literature survey shows that some results are already available to deal with a simplified approach of ship collisions, which would be time and cost-effective in the stage of predesigning large ships for example. This can be achieved by modeling the architecture of ships with very large-sized structural units and a limited number of nodal points. Using the literature references mentioned here above, closed-form analytical formulations of the resistance of each unit may be derived. Then, by combining properly the individual resistances, it is possible to obtain a global evaluation of the ability of a ship to withstand to an impact with another ship.

This basic idea was initially suggested by Ueda and Rashed [18], who called it the idealized structural unit method (ISUM). Lützen, Simonsen and Pedersen [26] developed a similar approach called the super-elements method (SEM), in which the ship is divided into large structural components (the socalled super-elements), whose resistance is assessed by making use of the above mentioned literature references. More recently, Le Sourne [19] coupled the super-elements method with a rigid body movement analysis tool in order to tackle both internal and external mechanics of a ship collision. The present paper is directly related to the super-elements method.

At the moment, this method is able to consider only collision scenarios which occur perpendicularly. The aim of the work presented in this article is to establish analytical expressions that can be used 
immediately to extend the procedure to oblique collision cases. The developed formulations are then validated by comparison with numerical simulations using finite element software. In fact, at these days, we don't have any result coming from physical oblique impacts performed in laboratories. As a consequence, the use of numerical comparison is the only validation process that can be used at this stage of innovative research.

\section{The super-elements method}

The super-elements method is a simplified approach to rapidly estimate the consequences of ship collision in a pre-design stage. The basic idea of the method is to evaluate the energy absorption involved during the penetration of the striking ship, by use of super-elements. This first aspect of the problem, often referred as the internal mechanics, has to be coupled with a dynamic solver to calculate the global ship motion (this second part of the problem is called the external mechanics). The internal and external mechanics are well described by Le Sourne [19] and are shortly summarized hereafter.

\subsection{The internal mechanics}

In the present paper, we admit that the bow of the striking ship is perfectly rigid. Consequently, internal mechanics is modeled by only dividing the struck ship into different structural components. These super-elements are assumed to behave independently of each other, i.e. there is no coupling between them. Under an impact, they will suffer important plastic deformations, for which it is possible to analytically estimate the associated dissipated energy.

In case of a right angle collision, according to Lützen [22], the architecture of the struck ship can be basically modeled with the four following super-elements:

- The first one is a rectangular plate simply supported on its four edges (Fig. 1). During a perpendicular impact, this plate will suffer large out-of-plane deflections dominated by a membranous behavior (see Zhang [6] for example). The rupture is supposed to occur when the deformations exceed a threshold value. Typically, this super-element is used to model inner and outer side plating and longitudinal bulkheads.

- The second one (Fig. 2) is a rectangular plate simply supported on three edges. The last edge is free and submitted to an in-plane load during a perpendicular collision. According to Simonsen and Ocakli [21], such an impacted plate will form successive folds until fracture. As suggested by Wierzbicki [12], the rupture occurs by tearing along the supported edges, which allows the plate to deform like a concertina. Typically, this super-element is used to model decks, transverse bulkheads, web girders, frames, bottom and inner-bottom.

- The third super-element (Fig. 3) is a beam loaded by a perpendicular transverse force. During a collision, it is supposed to collapse in two different phases. In the first step, it assumed that a plastic mechanism involving three plastic hinges occurs. After that, in a second step, the beam is behaving more like a plastic string. The rupture happens when the deformation reaches a critical value. This super-element is principally used to model small stiffeners like longitudinals.

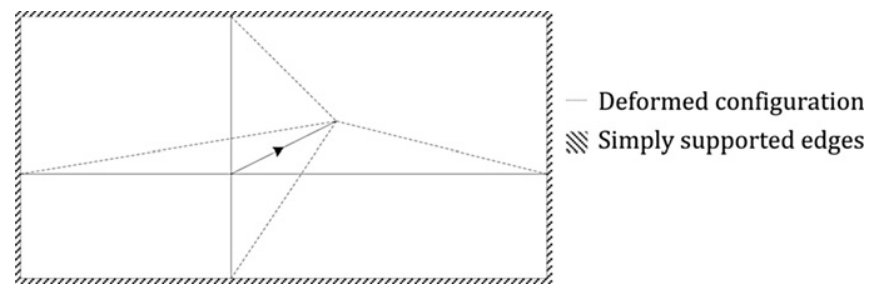

Fig. 1. Plate subjected to out-of-plane deformation. 


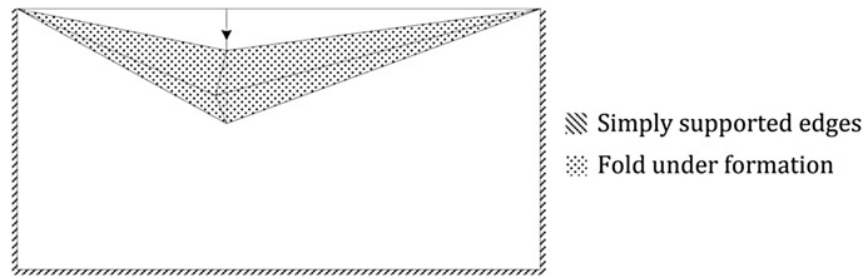

Fig. 2. Illustrations for the second super-element.

- The last super-element (Fig. 4) concerns X-T-L-form intersections. During a collision, they are assumed to be crushed axially, until they are completely deformed along their initial length. The rupture occurs by tearing of the plate on the supported edges. They are useful to model the junction of vertical and horizontal structural members.

With all the above super-elements is associated a closed-form expression which allows to calculate the energy dissipated by each of them during a perpendicular collision scenario. To obtain the total energy involved for a given penetration, it is sufficient to add the individual contribution of all the crushed super-element. This is a rather approximate method, because it neglects the interactions that may happen in reality between the various structural members. Nevertheless, comparisons with experiments and finite element simulations have shown a sufficiently good agreement (see [19,21] and Section 8), so that the method finally leads to a reasonable estimation of the struck ship resisting force $F$.

\subsection{The external mechanics}

After having evaluated the crushing force $F$ by means of the internal mechanics, the second task is to assess the energy released during the rigid global movement of the ships. This is the main purpose of the external mechanics. The method is extensively detailed in Refs. [6,19,20], so we will give here only a short presentation.

The external mechanics is based on a classical rigid-body theory. The principal useful equation is the second Newton's law. If we refer to Fig. 5, the equations of motion are:

$$
\left[M_{1}\right] \dot{V}_{1}=R_{1} \quad\left[M_{2}\right] \dot{V}_{2}=R_{2}
$$

where

$\left[M_{1}\right]$ and $\left[M_{2}\right]$ are the structural mass matrix associated respectively to ship 1 and 2

$\dot{V}_{1}$ and $\dot{V}_{2}$ are the velocities of ship 1 and 2

$R_{1}$ and $R_{2}$ are the contact and hydrodynamic forces acting on ship 1 and 2

To apply these equations, it is required to know all the forces acting on the collided ships. According to [19], we have to consider:

- The contact loads $F_{\mathrm{c}}$ (forces and resulting moments) exerted by both ships and calculated at their center of gravity.

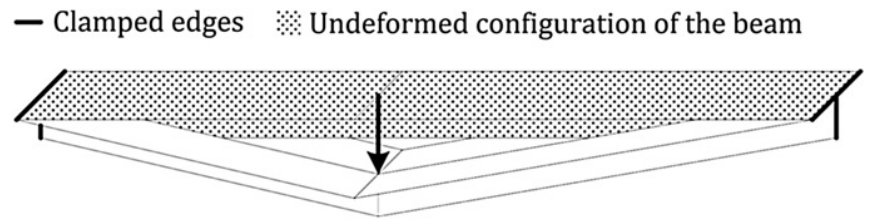

Fig. 3. Deformation of an impacted beam with a $\mathrm{T}$ cross-section. 

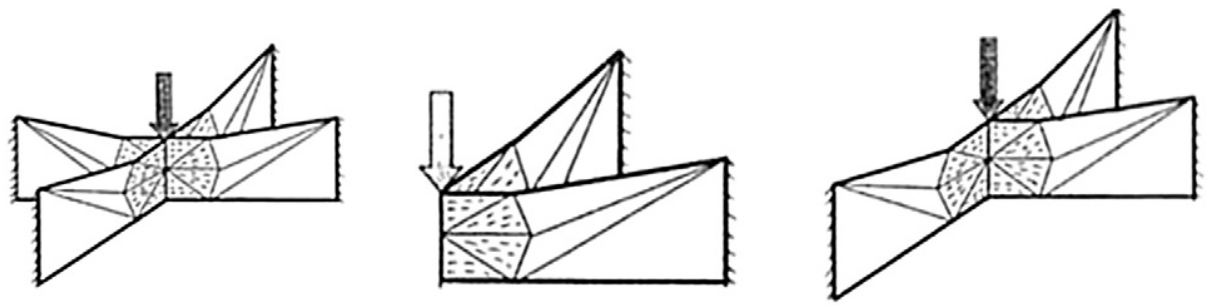

Fig. 4. Deformation mechanisms of $\mathrm{X}, \mathrm{L}$ or $\mathrm{T}$ intersections - the figures are extracted from [6].

- The hydrodynamic forces and moments due to the surrounding water. These forces and moments have generally different contributions, such as buoyancy $F_{b}$, gravity $F_{g}$, viscosity $F_{v}$, waves $F_{w}$ and inertia, which is taken into account by an added mass matrix $\left[M_{\mathrm{a}}\right]$.

Consequently, equation (1) may be rewritten by making use of the previously described notations:

$$
\begin{aligned}
& \left(\left[M_{1}\right]+\left[M_{a 1}\right]\right) \dot{V}_{1}=F_{c 1}+F_{b 1}+F_{g 1}+F_{v 1}+F_{w 1} \\
& \left(\left[M_{2}\right]+\left[M_{a 2}\right]\right) \dot{V}_{2}=F_{c 2}+F_{b 2}+F_{g 2}+F_{v 2}+F_{w 2}
\end{aligned}
$$

The goal of this article is not to describe in details all the analytical expressions useful for the evaluation of each term involved in equation (2). For the purpose of this paper, it is sufficient to remember that (2) provides a good way to estimate the temporal evolution of $\dot{V}_{1}$ and $\dot{V}_{2}$.

\subsection{Ship collision analysis procedure}

To proceed to the analysis of ship collision, it is first necessary to collect information on the structural design of the struck section. It is also required to define the collision scenario (initial velocities, impact location, collision angle...) before starting any calculation. As soon as these parameters are defined, the crushing resistance of the struck ship can be evaluated simply by following the flow chart presented in Fig. 6, which is coherent with the assumption of a nondeformable bow.

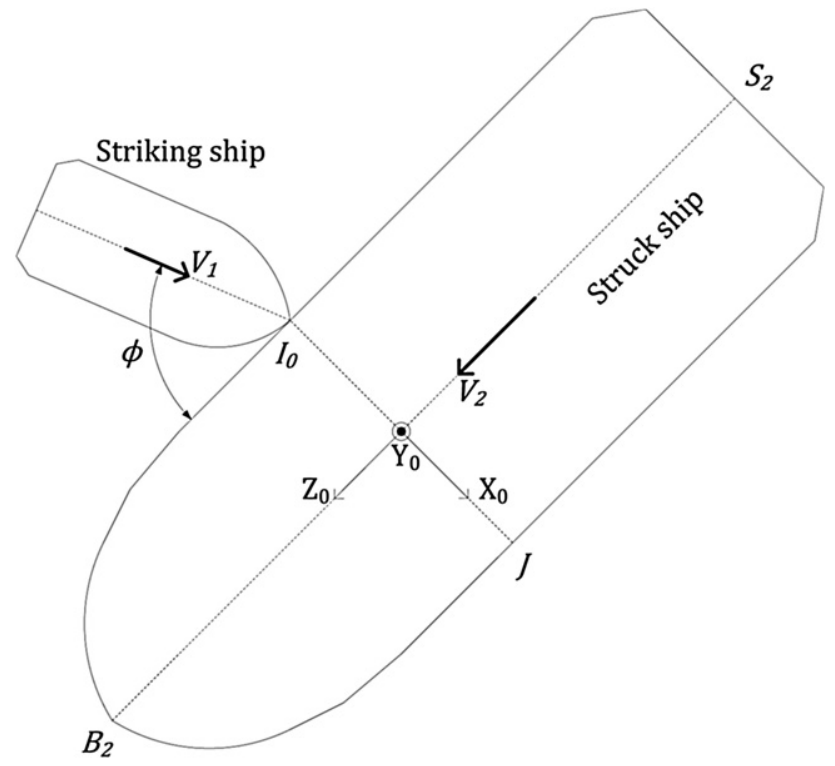

Fig. 5. Definition of velocities $V_{1}$ and $V_{2}$ of the two ships. 


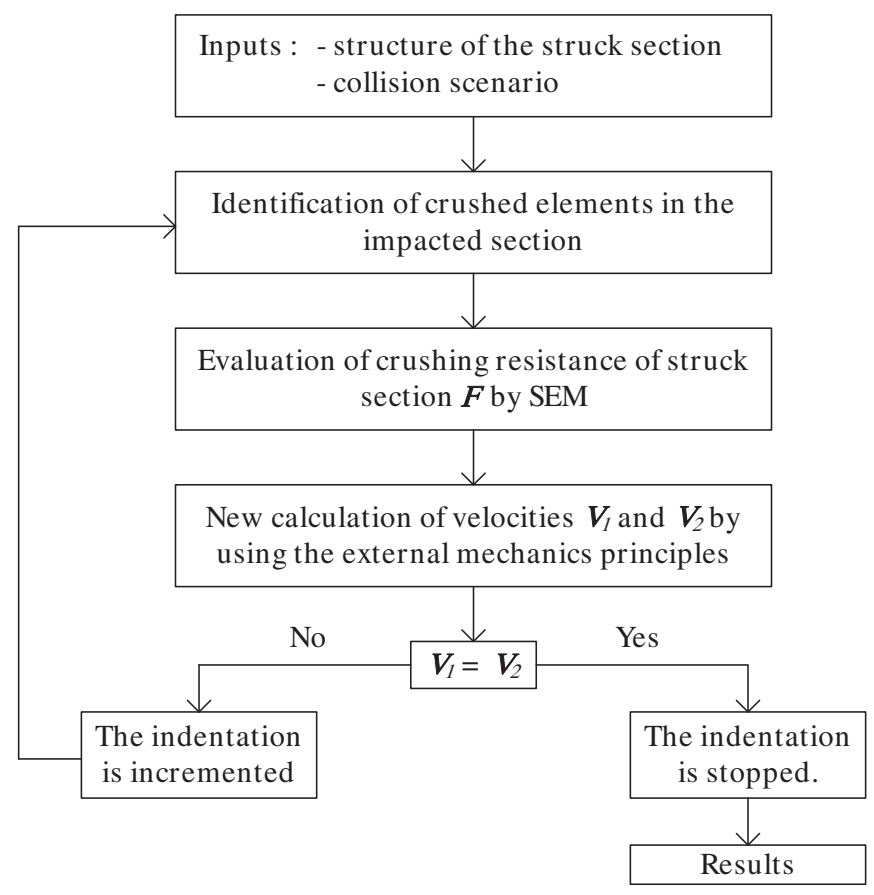

Fig. 6. Flow chart of the ship collision analysis procedure.

At this stage, it is important to note that the components detailed above are sufficient while dealing only with right angle collisions. However, for the more general case of oblique collision, it will be required to develop other types of super-elements.

\section{Introduction to the analysis of oblique collisions}

The super-elements developed originally for perpendicular collisions are insufficient for treating the case of impact with an oblique angle. Therefore, it is required to develop new super-elements, or adapt the existing one.

\subsection{Theoretical basis}

Before detailing each super-element, it is necessary to describe the method leading finally to the evaluation of the resistance in the oblique collision case. The fundamental basis is the so-called "upperbound theorem" which, according to Jones [24], states that "if the work rate of a system of applied loads during any kinematically admissible collapse of a beam is equated to the corresponding internal energy dissipation rate, then that system of loads will cause collapse of the structure".

Mathematically, during an impact, the maximal force $F$ responsible of the collapse of a given superelement with volume $V$ may be obtained by:

where

$$
F \cdot \dot{\delta}=\iiint_{V} \sigma_{i j} \cdot \dot{\varepsilon}_{i j} \cdot d V
$$

$\dot{\delta}$ is the penetrating speed of the striking ship in the super-element $\sigma_{\mathrm{ij}}$ is the stress tensor of the super-element 
$\dot{\varepsilon}_{\mathrm{ij}}$ is the strain rate tensor of the super-element

In order to obtain a closed-form expression of the collision resistance, some simplifications have to be made, unless equation (3) would be too complicated to be solved analytically. For purpose of simplicity, the following hypotheses are made:

- the material of the element is assumed to be perfectly rigid, as shown on Fig. 7. By doing such hypothesis, the elastic strains, the strain hardening and the strain rate effect are neglected.

- the total internal energy rate is obtained by summing the contribution of bending and membrane effects, which are assumed to be completely uncoupled.

For a plate in a plane-stress state, if we assume that bending effects are confined in a certain number $m$ of plastic hinge lines, we can calculate the bending $\dot{E}_{b}$ and membrane $\dot{E}_{m}$ energy rates with help of these two formulas:

$$
\dot{E}_{b}=M_{0} \sum_{k=1}^{m} \dot{\theta}_{k} l_{k} \quad \dot{E}_{m}=\frac{2 \sigma_{0} t_{p}}{\sqrt{3}} \iint_{A} \sqrt{\dot{\varepsilon}_{11}^{2}+\dot{\varepsilon}_{22}^{2}+\dot{\varepsilon}_{12}^{2}+\dot{\varepsilon}_{11} \dot{\varepsilon}_{22}} \cdot d A
$$

where $M_{0}$ is the fully plastic bending moment, $A$ and $t_{p}$ are the area and the thickness of the plate, $\theta_{k}$ and $l_{k}$ are the rotation and the length of hinge number $k$.

The most difficult part in the equation above remains the calculation of the strain rate tensor. This has to be done by choosing displacements fields, which are close enough with those observed on impact trials or on numerical simulations (in absence of tests). The problem with the upper-bound method is that it can overestimate the resistance if the displacements fields are not in good accordance with reality. However, the purpose of the following sections is to apply this method to evaluate the individual crushing resistance of several super-elements in the case of non-perpendicular impacts.

\subsection{General overview of the problem}

In order to deal successfully with oblique collisions, six different super-elements (denoted by SE1 to SE6) have been developed. Before describing them, we first need to define more precisely the problem already depicted on Fig. 5 and shown with more details in Fig. 8.

In this paper, we consider that a ship with initial velocity $\dot{V}_{1}$ impacts another ship with initial velocity $\dot{V}_{2}$. The collision happens at certain point $I_{0}$ with a given angle $\phi$ (which may be different from $\left.90^{\circ}\right)$. For sake of clarity, it is important to note that a unique global reference frame $\left(X_{0}, Y_{0}, Z_{0}\right)$ is defined in Fig. 5. Its origin is located in the uppermost deck of the struck ship, at the intersection between:

- the symmetry line $\mathrm{B}_{2} \mathrm{~S}_{2}$ joining the bow and the stern of the struck ship;

- the impact line $\mathrm{I}_{0} \mathrm{~J}$, defined by the initial contact point $I_{0}$ and its inverse $J$.



Fig. 7. Example of stress-strain curve for mild steel. 


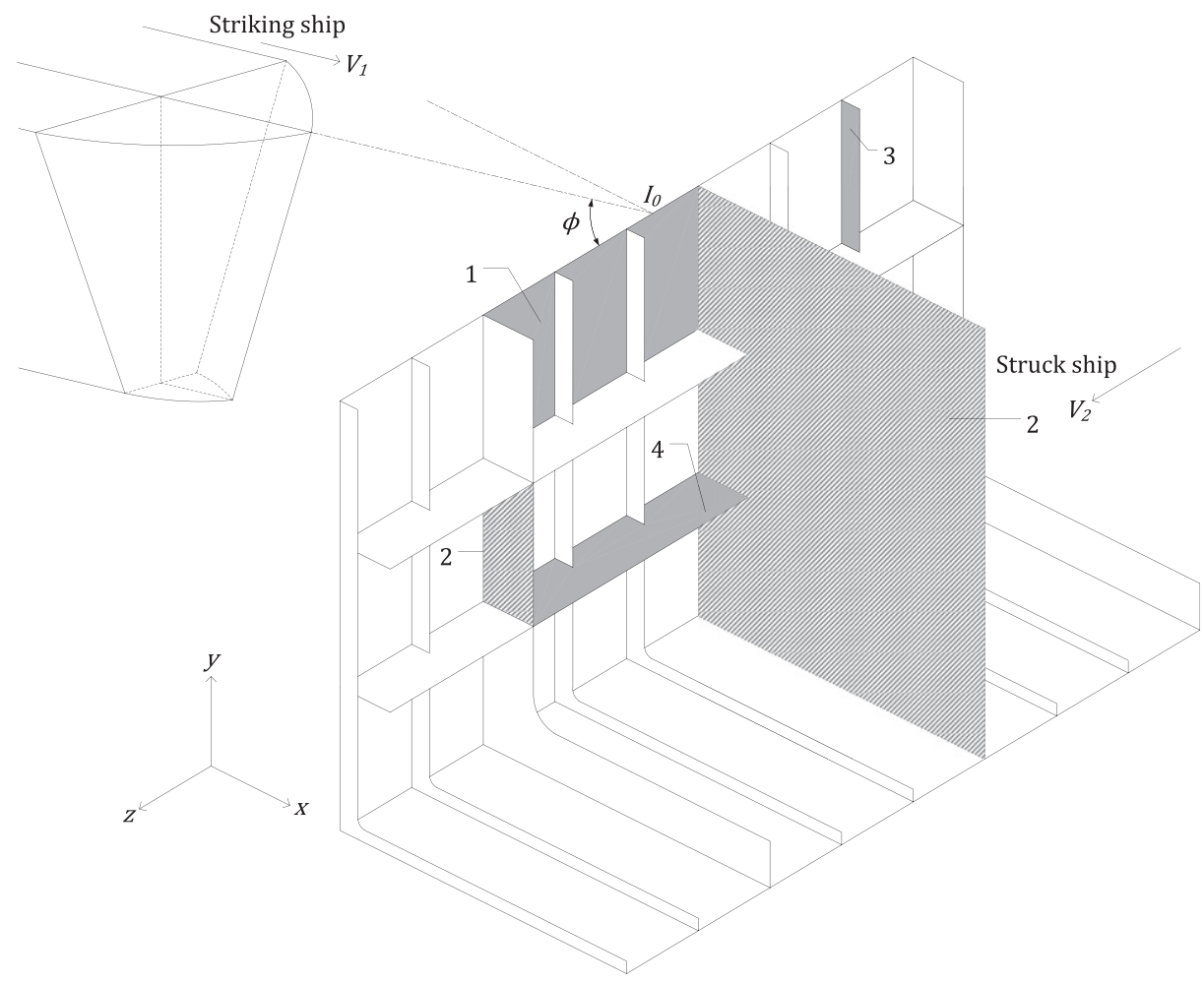

Fig. 8. Description of the collision scenario.

The vertical $Y_{0}$ axis is pointing upward, the first horizontal $Z_{0}$ axis is oriented with the same direction than the velocity $\dot{V}_{2}$, while the second horizontal $X_{0}$ axis is defined for obtaining an adequate $\left(X_{0}, Y_{0}, Z_{0}\right)$ right-handed reference frame. However, while dealing with individual super-element, it is sometimes more convenient to use a local reference frame $(x, y, z)$, whose axes are always defined such that $x\left\|. X_{0}, y\right\| . Y_{0}$ and $z \| . Z_{0}$ (the axes are parallel to those of the global frame, but the origin is located anywhere in the space). Now that the problem is exposed, we can describe each super-element individually. This is precisely the purpose of the following sections. However, in this paper, we won't treat the so-called "super-element 5", because it deals with the impact on X-T-L-form intersections (see Fig. 4). As the extension to oblique collisions implies very little modifications in comparison with the work performed by Amdahl [17] and Zhang [6], it is not relevant to describe them here again.

\section{Description of super-element 1}

\subsection{Analytical model}

This first super-element (SE1), represented on Fig. 9, is used to assess the resistance of a plate simply supported on its four edges, and submitted to an out-of-plane impact occurring with a certain angle $\phi$, which may be different from $90^{\circ}$. In order to have clearer representation, this super-element is also illustrated by the plate designated by label 1 in Fig. 8 .

At the beginning, when the penetration $\delta$ of the striking vessel is still equal to 0 , the bow impacts the super-element at point $I_{0}$ (see Fig. 10). The plate is then divided into four zones (numbered I-IV in Fig. 10), which are initially defined in accordance with Table 1 , for $\delta=0$. However, for a given 


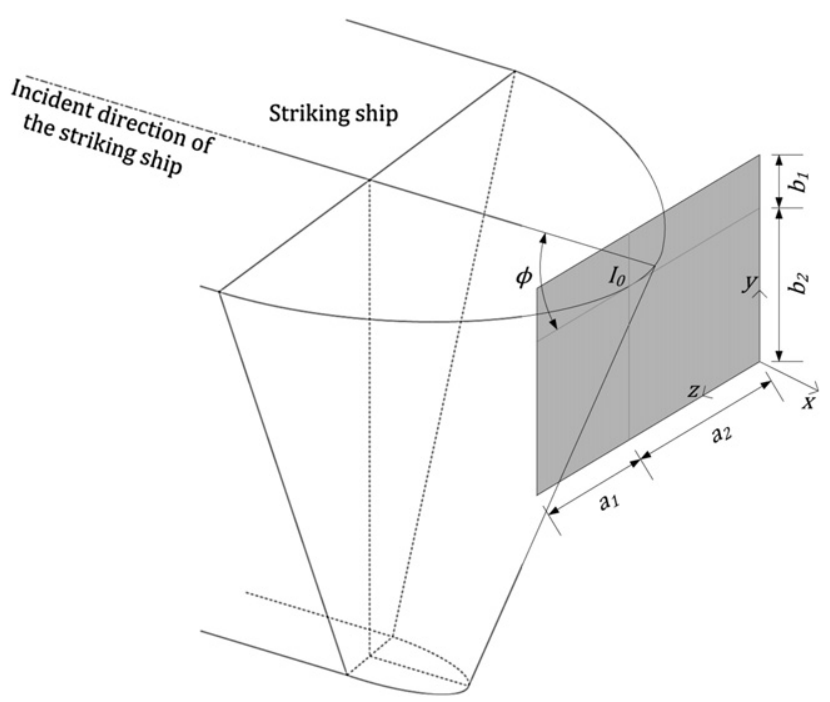

Fig. 9. General representation of the collision scenario for super-element 1.

penetration $\delta>0$, the contact point is moving from its initial position $I_{0}$ to the current one $I$ (see Fig. 11), and we have $\delta=I_{0} I$. By denoting $I^{\prime}$ the orthogonal projection of point $I$ on the plate, it is possible to update the definition of surfaces I-IV (see Table 1).

For these four surfaces, a kinematically admissible displacements field $w(y, z)$ is defined and oriented along the perpendicular direction to the plane of the plate (i.e. along $x$ axis on Figs. 10 and 11). For example, considering surface I, we have:

$$
w(y, z)=\left(\frac{z}{a_{1}+\delta \cos \phi}\right)^{n}\left(\frac{y}{b_{1}}\right)^{n} \delta \sin \phi
$$

where $n$ is a natural number that may be arbitrary chosen for having a close agreement between numerical and analytical results (see Section 4.2). This can be achieved by taking $n=2$. However, for values of $n$ greater than 2 , there is little change in the displacement profile given by (5), so the
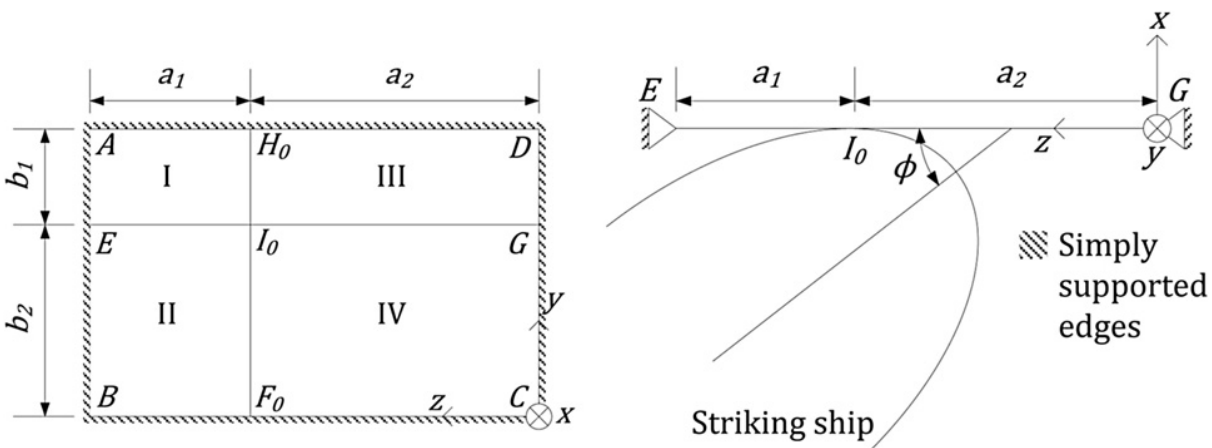

Fig. 10. Initial dispositions at the beginning of the collision. 
Table 1

Definition of surfaces I-IV for super-element 1.

\begin{tabular}{|c|c|c|c|c|c|c|c|}
\hline \multicolumn{4}{|c|}{ Initial configuration $(\delta=0)$} & \multicolumn{4}{|c|}{ Deformed configuration $(\delta>0)$} \\
\hline Surface I & Surface II & Surface III & Surface IV & Surface I & Surface II & Surface III & Surface IV \\
\hline $\mathrm{AEI}_{0} \mathrm{H}_{0}$ & $\mathrm{EBF}_{0} \mathrm{I}_{0}$ & $\mathrm{H}_{0} \mathrm{I}_{0} \mathrm{GD}$ & $\mathrm{I}_{0} \mathrm{~F}_{0} \mathrm{CG}$ & $\mathrm{AEI}^{\prime} \mathrm{H}$ & EBFI' $^{\prime}$ & $\mathrm{HI}^{\prime} \mathrm{GD}$ & $\mathrm{I}^{\prime} \mathrm{FCG}$ \\
\hline
\end{tabular}

correlation between numerical and analytical results is not significantly improved. In order to verify the compatibility at the junction between each surface, this field has to be completed by the four plastic hinges HI, EI, FI and GI (see Fig. 11).

With help of equation (5), it is now possible to evaluate the strain rates tensor for each of the four surfaces I-IV. This can be achieved immediately by applying Green's formulas. The mathematical expressions of the strain rates are given by equations (22)-(24) in Appendix 1.1. The deformation rates may then be introduced in equation (4) in order to obtain the total membrane energy $\dot{E}_{m}$ absorbed by super-element 1 (more details on the analytical procedure are presented by relations (25)-(29) in Appendix 1.1). However, as the plate thickness is often reasonably small, the contribution of bending $\dot{E}_{b}$ in the four above-mentioned plastic hinges may be neglected in comparison with $\dot{E}_{m}$. So the total internal energy rate is simply given by $\dot{E}_{m}$.

Let's now denote by $F_{x}$ and $F_{z}$ the components of the crushing force acting respectively along $x$ and $z$ axis (see Fig. 11), the application of the virtual work principle leads to:
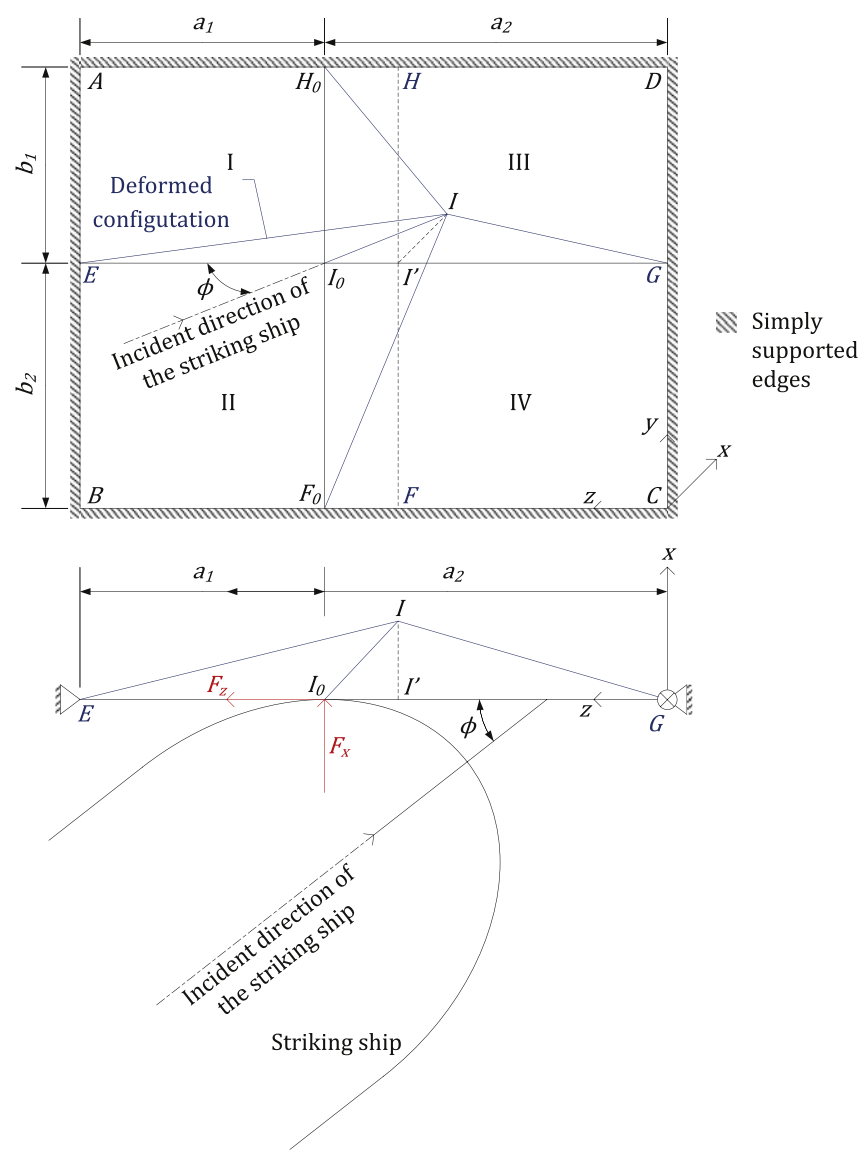

Fig. 11. Deformed configuration of plate during the collision. 


$$
\dot{E}_{m}=F_{x} \cdot \dot{\delta} \sin \phi
$$

Under the assumption that the force $F_{Z}$ acting in the plane of the plate is only coming from the friction between the impacted structure and the striking ship, we have $F_{z}=\mu F_{\mathrm{x}}$, where $\mu$ is the Coulomb friction coefficient of the material. The crushing resultant force $F$ of super-element 1 is then given by:

$$
F=\sqrt{F_{x}^{2}+F_{z}^{2}}=F_{x} \cdot \sqrt{1+\mu^{2}}=\frac{\dot{E}_{m}}{\dot{\delta} \sin \phi} \sqrt{1+\mu^{2}}
$$

with $\dot{E}_{m}$ given by relation (29). Equation (7) gives the evolution of the crushing resistance with respect to the indentation $\delta$, for a certain angle $\phi$. Of course, this law remains valid as long as there is no rupture in the structure. This is assumed to happen when the effective plastic strain $\varepsilon$ reaches a critical value $\psi$ somewhere in the plate. In accordance with Zhang [6], Lützen [22] or Brown [25], $\psi$ should be chosen within a range of $5-12 \%$ for a steel-like material. As soon as $\varepsilon \geq \psi$, the crushing resistance of the plate is set to 0 . For this super-element, we have chosen $\varepsilon=10 \%$.

\subsection{Numerical validation}

In order to validate the analytical formulation developed here above, some numerical simulations have been performed by using the non-linear finite element code LS-DYNA. As numerical data used for validation, the parameters listed in Table 4 (see Appendix 1.2) have been chosen.

Finite element models of the striking ship and of the impacted plate are shown on Fig. 12 (for clarity, the scale between the bow and the plate is not respected), where axis $(x, y, z)$ and angle $\phi$ are also indicated to have a better localization of the collision situation in the space. Total dimensions of the plate are $6 \mathrm{~m} \times 8 \mathrm{~m}$, which may be quite surprising while dealing with structural components of ship. However, it is important to recall here that super-element 1 corresponds to the complete side panel located between transverse bulkheads or web girders (see number "1" on Fig. 8 for example). Such panels may of course be reinforced by some stiffeners, but those are treated separately (see Section 6). In the present case, our simulations used 20,650 Belytschko-Tsay shell elements [27] for the striking ship and 19,200 similar shell elements for the impacted structure, which corresponds to a quite refined mesh size of $5 \mathrm{~cm} \times 5 \mathrm{~cm}$. Moreover, the plate is modeled as simply supported on its four edges.

The numerical material law used for the striking bow is assumed to be perfectly rigid. For the struck plate, we used a numerical material law that represents more or less the behavior of steel (see Appendix 1.3 for more information). Rupture of the plate happens at a particular critical deformation $\varepsilon_{\mathrm{c}}=25 \%$. When this failure level is reached, the corresponding finite element is deleted from the model. We know that it is still difficult to deal properly with rupture using finite element method: even if some special material models using element length-dependent failure strain have been developed (see $[29,30]$ for example), but they only have been implemented as usermat laws and are not yet available in the LS-DYNA version used for this study. Therefore, the value of $25 \%$ has been chosen in accordance with the work performed by Le Sourne [28], where different failure criteria were adapted to different mesh sizes for various deformation modes (membrane tension, bending and tearing).

Fig. 13 represents a comparison between analytical and numerical results, while considering the parameters reported in Table 4. It can be seen that the agreement is satisfactory. The value $\psi=10 \%$ seems also to be a reasonable choice to simulate the rupture of the steel constituting the plate.

\section{Description of super-elements 2 and 3}

For super-elements 2 and 3, the impacted structure is a plate supported on three edges, the last one remaining free. They differ from each other by the impact location. For SE2, the impact is assumed to be located on the unsupported side (see Fig. 15a), while for SE3, the contact point is located somewhere inside the plate (see Fig. 15b). It is important to distinguish between these two situations because the displacement fields are not the same in both cases. However, as these elements exhibits some 


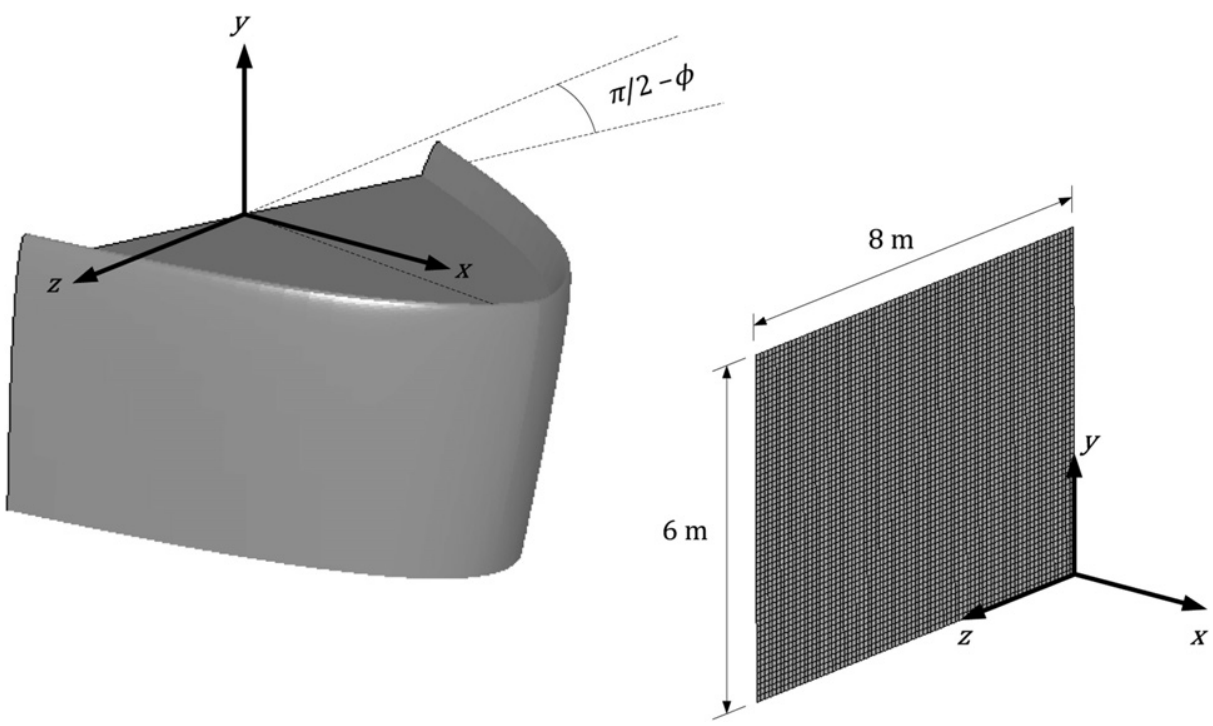

Fig. 12. Finite element modeling of the striking ship and the impacted plate.

similarities, we have decided to treat them in a unique section. Typically, they are used for modeling transversal bulkheads (see the components designated by label 2 in Fig. 8).

\subsection{Analytical model}

When the impact happens on the free edge, the initial contact point $I_{0}$ allows for the definition of the two main dimensions $a_{1}$ and $a_{2}$ (see Fig. 14a). When the ship is moving forward, the contact point $I$ doesn't follow the incident direction of the striking ship but is rather positioned at a given place along the stem (see Fig. 16b). The deformation pattern is then as shown on Fig. 14b. It is composed of two plastic hinge lines, denoted by BD and BG, and two related deforming triangular surfaces IBD and IBG where only membrane deformations occur. To completely define this deformation mechanism, it is necessary to fix points $B$ and $I$. The first one is simply taken as the intersection between the stem and the $x$ axis (see Figs. 14b and 16b), while the second is located somewhere on the striking ship stem such that:

$$
\overline{B I}_{0}=\overline{B I}
$$

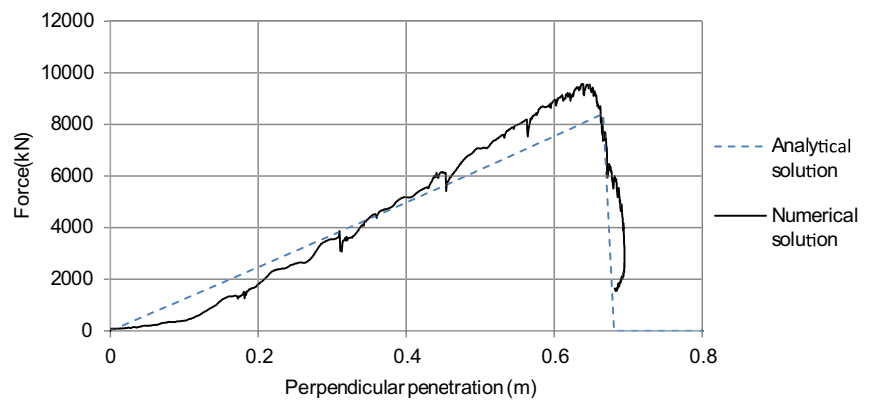

Fig. 13. Comparison between numerical and analytical solution for SE1. 

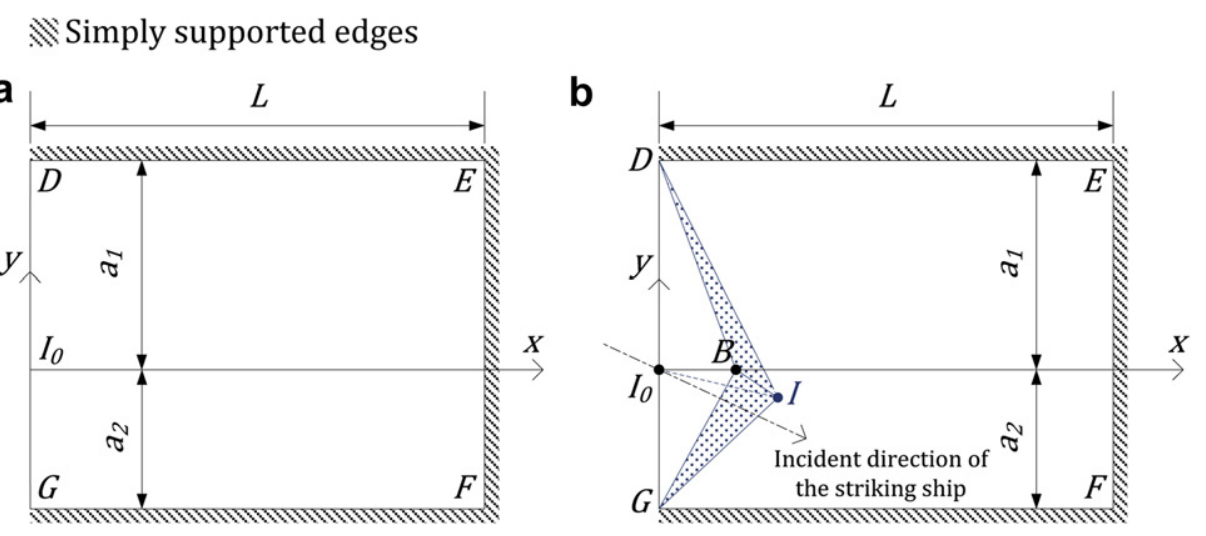

Fig. 14. Initial (a) and deformed (b) configurations of SE2 in the plane of the impacted plate.

In other words, using equation (8), we made the assumption that the plate is rotating around two plastic hinges $\mathrm{BD}$ and $\mathrm{BG}$ without any deformation along the $x$ direction. For this reason, the fibers belonging to surfaces $\mathrm{I}_{0} \mathrm{BD}$ and $\mathrm{I}_{0} \mathrm{BG}$ are only submitted to an axial extension along the $y$-axis.

Consequently, we just have to define a displacement field $v(x, y)$ oriented along this axis (see Appendix 2.1 for more details). We have:

$$
\begin{array}{ll}
\text { For surface } I_{0} B D & v(x, y)=\frac{1}{2} \cdot \frac{p^{2}}{a_{1}} \cdot \frac{b-x}{b} \cdot \frac{a_{1}-y}{a_{1}} \\
\text { For surface } I_{0} B G & v(x, y)=\frac{1}{2} \cdot \frac{p^{2}}{a_{2}} \cdot \frac{b-x}{b} \cdot \frac{a_{2}-y}{a_{2}}
\end{array}
$$

where $p=\overline{I_{0} I}$ and $b=\overline{I_{0} B}$ (see Fig. 14b). With equation (9) and by application of Green's formulas, it is possible to evaluate the membrane energy $E_{m}$ dissipated by surfaces $\mathrm{I}_{0} \mathrm{BD}$ and $\mathrm{I}_{0} \mathrm{BG}$. This is the first contribution to the total energy dissipated by the structure during the impact.

Another contribution to the internal energy arises from the rotations taking place in the plastic hinge lines BD and BG. The associated bending energy $E_{b}$ may be evaluated with help of the rotation
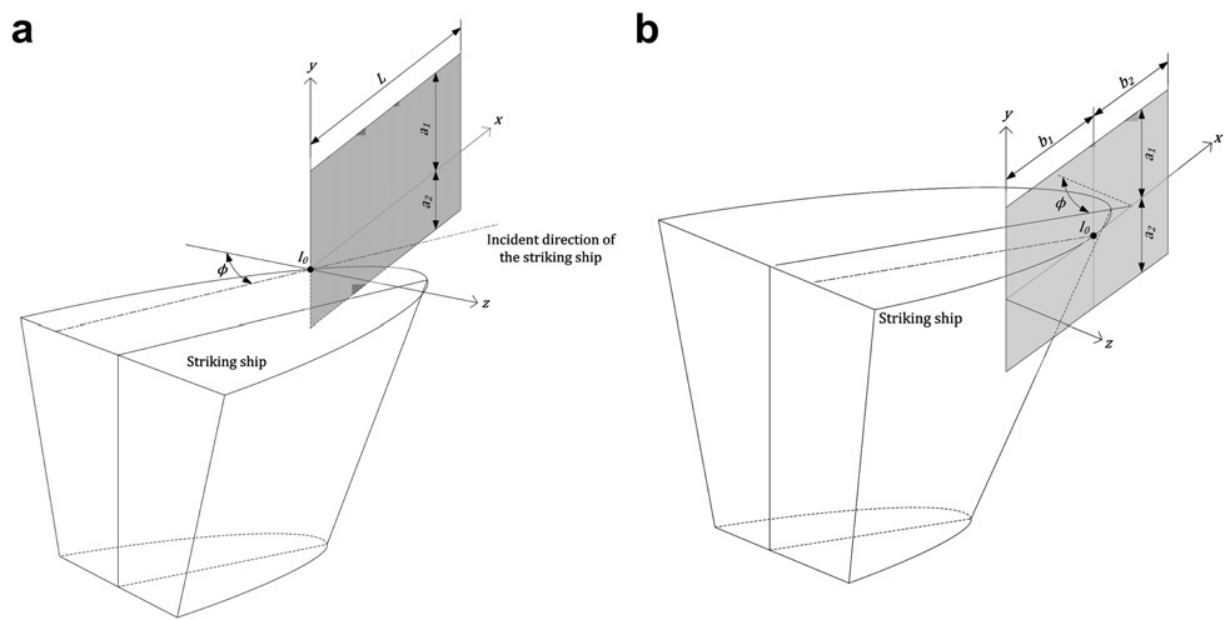

Fig. 15. (a) Collision scenario for super-element 2; (b) Collision scenario for super-element 3. 

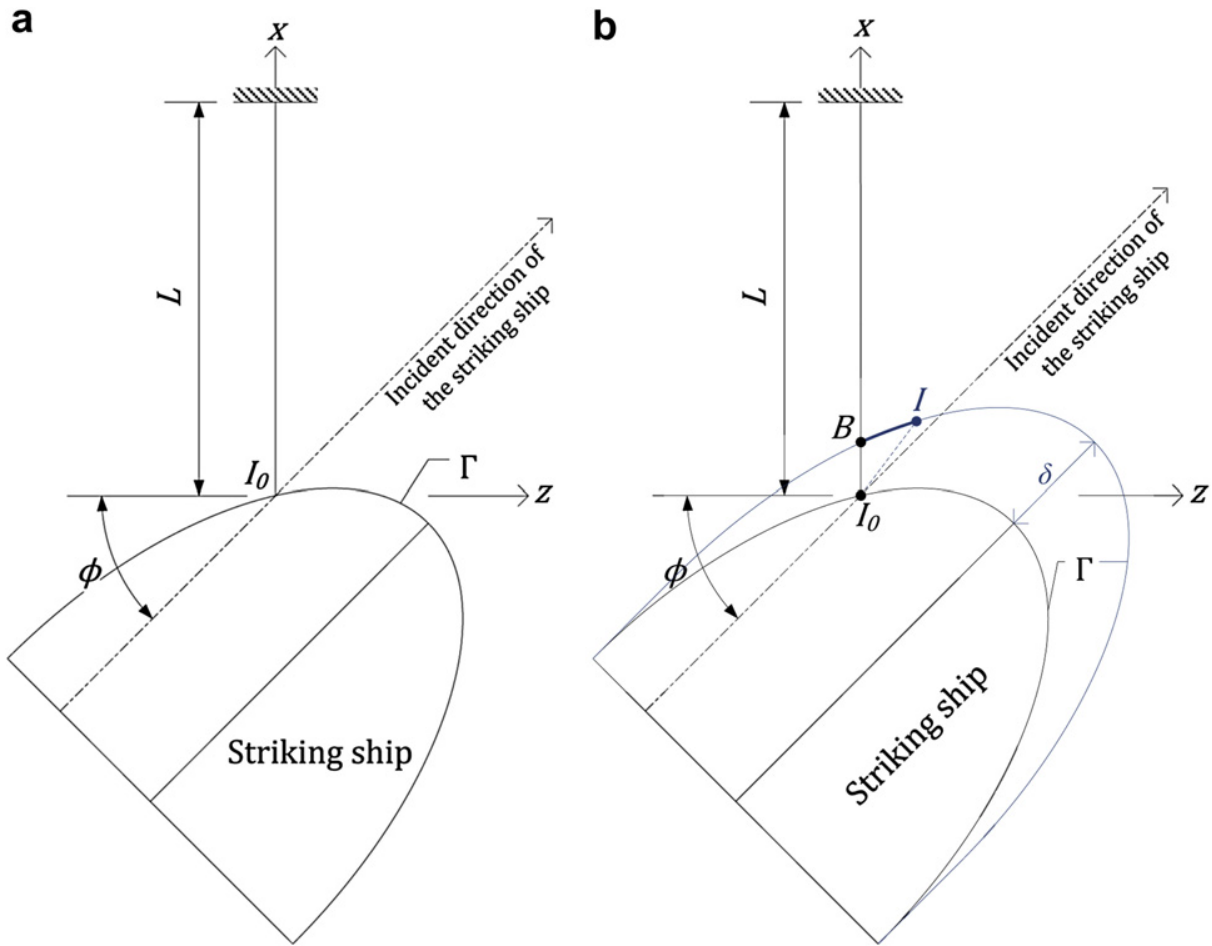

Fig. 16. Top view of the collision at the beginning of the impact (a) and for a given penetration (b).

angles $\theta_{1}$ and $\theta_{2}$ respectively made by surfaces IBG and IBD with the plane $(x, y)$. Some geometrical considerations (see Appendix 2.1) show that we have:

$$
\begin{aligned}
& \text {-For surface } I_{0} B D \quad \theta_{1}=\arccos \left(\frac{a_{1}\left(2 b^{2}-p^{2}\right)}{2 b^{2}-\sqrt{a_{1}^{2}+b^{2}}}\right) \\
& \text {-For surface } I_{0} B G \quad \theta_{2}=\arccos \left(\frac{a_{2}\left(2 b^{2}-p^{2}\right)}{2 b^{2}-\sqrt{a_{1}^{2}+b^{2}}}\right)
\end{aligned}
$$

Equation (10) allows for the evaluation of the energy $E_{b}$ dissipated by bending in the plastic hinges $\mathrm{BD}$ and BG. Then, by applying the virtual velocities principle (see details in Appendix 2.1), a closed-form expression of the SE2 crushing resistance is obtained:

$$
F=\frac{\sigma_{0} t_{p}}{2} \cdot \frac{a_{1}+a_{2}}{a_{1} a_{2}} \cdot p\left(b \frac{\partial p}{\partial \delta}+\frac{p}{2} \frac{\partial b}{\partial \delta}\right)+\frac{\sigma_{0} t_{p}^{2}}{4}\left(\sqrt{a_{1}^{2}+b^{2}} \frac{\partial \theta_{1}}{\partial \delta}+\sqrt{a_{2}^{2}+b^{2}} \frac{\partial \theta_{2}}{\partial \delta}\right)
$$

where $a_{1}$ and $a_{2}$ are the distances between the impact point and the fixed edges (see Fig. 14) and $t_{p}$ is the plate thickness. Parameters $b$ and $p$ have been defined previously, according to the location of points $B$ and $I$. Their mathematical expressions can be derived by assuming that the curve $T$ describing the plane shape of the bow on Fig. 16 is an ellipse, which is acceptable for most ships. Unlike other super-elements, it is important for SE2 and SE3 to account for the shape of the striking ship, because numerical simulations have shown that the deformed profile of the impacted structure is closely related to the shape of the bow. This is mainly due to the fact that the plate is not constrained along the impacted edge, which allows it to "follow" more closely the curve designated by $\Gamma$ on Fig. 16. As 
a consequence, it is no longer possible to assume that the displacement field is only governed by a punctual contact, as it is done for the other super-elements.

Finally, as for SE1, we still have to distinguish between the behavior before and after failure. For a critical penetration $\delta_{\mathrm{c}}$, the deformation becomes larger than a threshold value $\psi$ and rupture happens. However, it would be over-conservative to simply set the resistance to zero as it has just been done for SE1. In fact, rupture of SE2 occurs by tearing along the supported edges, which results in a decrease of the crushing resistance. For a given penetration $\delta_{\mathrm{f}}$, the tearing length is sufficient to allow the striking ship to bypass the super-element. The resistance has then to be set to zero. For $\delta_{\mathrm{c}} \leq \delta \leq \delta_{\mathrm{f}}$, the resistance is assumed to decrease linearly.

The following part of the present section will now be devoted to a short presentation of superelement 3. This time, the initial contact point $I_{0}$ is located at a distance $b_{1}$ of the unsupported edge GD (see Fig. 17a). As it was done previously for SE2, for a given penetration $\delta$, this point is moving along the elliptical shape of the striking bow (Fig. 18) to reach its current position $I$. If we define point $B$ in a similar manner than for SE2, it is still possible to locate point $I$ by respecting condition (8). Consequently, on Fig. 17b, the deformed configuration IBD and IBG of the two triangular surfaces $\mathrm{I}_{0} \mathrm{BD}$ and $\mathrm{I}_{0} \mathrm{BG}$ is determined in a similar manner than the one depicted on Fig. 14a. However, we still have to consider the remaining part of the plastic mechanism, i.e. the two triangles $\mathrm{I}_{0} \mathrm{HD}$ and $\mathrm{I}_{0} \mathrm{HG}$. This additional development was not required for SE2 as the impact was directly located on the free edge, so that we had $b_{1}=0$.

During the impact, the current configurations of surfaces $\mathrm{I}_{0} \mathrm{HD}$ and $\mathrm{I}_{0} \mathrm{HG}$ are given by IJD and IJG. For completely defining this deformation process, we made the following hypotheses:

- For current values of the plate thickness $t_{p}$, the plastic bending capacity of the plate $\left(\sigma_{0} t_{\mathrm{p}}^{2} / 4\right)$ is quite small, which means that the bending energy $E_{\mathrm{b}}$ may be neglected in comparison with the membrane energy $E_{m}$. This conservative assumption was not made in the case of SE2, but we have decided to make it here for simplicity.

- There is no axial straining along $x$ direction, which means that the segment $\mathrm{HI}_{0}$ always keeps its original length $b_{1}$ and requires that $\overline{H J}=\overline{I_{0} I}$. This is justified by the fact that the free edges HD and HG are not able to exert an important translational restrain on the horizontal fibers. Consequently, the membrane energy dissipated during the deformation is entirely produced by an axial extension of the fibers parallel to $y$-axis.

- The connection between IJD and IBD (or between IJG and IBG) is made by the plastic hinge line ID (or IG) allowing for a relative rotation of this two triangles. If this was not the case, the previous assumption would necessarily lead to a non-kinematically admissible displacement field.

As a consequence of the previous hypotheses, the deformed configuration of SE3 for a given penetration $\delta$ is reached by imposing an axial extension of the fibers parallel to $y$-axis and a rotational

NSimply supported edges
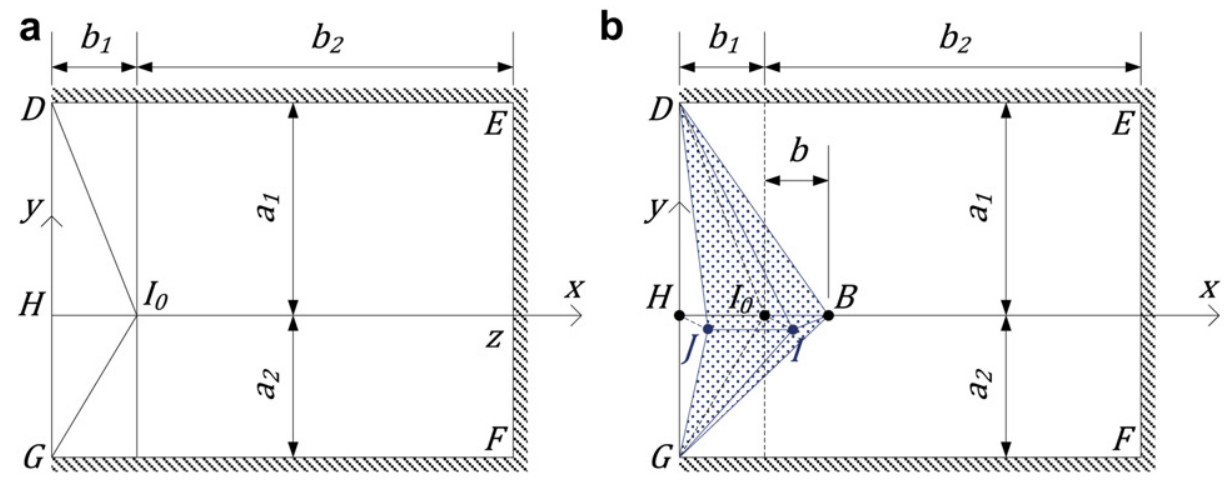

Fig. 17. Initial (a) and deformed (b) configurations of SE3 in the plane of the impacted plate. 
a

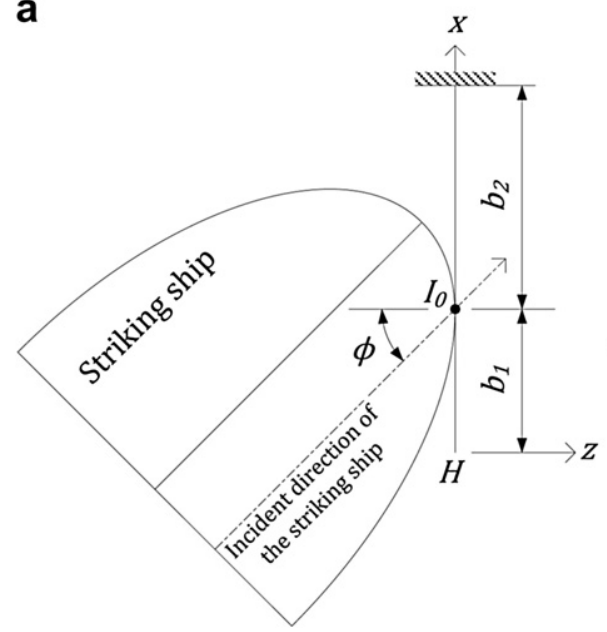

b

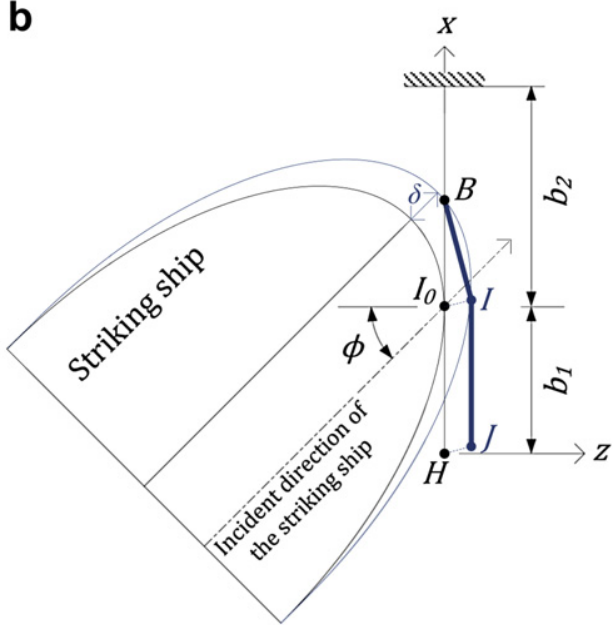

Fig. 18. Top view of the collision at the beginning of the impact (a) and for a given penetration (b).

motion around the plastic lines ID, IG, BD and BG. As we only deal with membrane effects, we just have to consider an axial displacement field $v(x, y)$ which is derived in a similar manner than for SE2:

$$
\begin{aligned}
\text {-For surface HBD } & \text { if } x \leq b_{1}: \quad v(x, y)=\frac{p^{2}}{2 a_{1}} \frac{b_{1} x+b^{2}-x^{2}}{b^{2}} \cdot \frac{a_{1}-y}{a_{1}} \\
& \text { if } x>b_{1}: \quad v(x, y)=\frac{p^{2}}{2 a_{1}} \frac{b-x+b_{1}}{b} \frac{a_{1}-y}{a_{1}} \\
\text {-For surface HBD } & \text { if } x \leq b_{1}: \quad v(x, y)=\frac{p^{2}}{2 a_{2}} \frac{b_{1} x+b^{2}-x^{2}}{b^{2}} \cdot \frac{a_{2}-y}{a_{2}} \\
& \text { if } x>b_{1}: \quad v(x, y)=\frac{p^{2}}{2 a_{2}} \frac{b-x+b_{1}}{b} \frac{a_{2}-y}{a_{2}}
\end{aligned}
$$

where $p=\overline{I_{0} I}$ and $b$ are used for locating the current position of $B$ (see Fig. 17b), as it was done for SE2. By using (12) in Lagrange's equations, it is possible to evaluate the deformation $\varepsilon_{\mathrm{yy}}$ and the membrane energy rate. As bending is not considered here, this procedure leads finally to the following expression of the crushing force:

$$
F=\frac{\sigma_{0} t_{p}}{6 b} \cdot \frac{a_{1}+a_{2}}{a_{1} a_{2}} \cdot \frac{p}{b}\left(\left(3 b^{3}+6 b^{2} b_{1}+b_{1}^{3}\right) \frac{\partial p}{\partial \delta}+\left(3 b^{3}-2 b_{1}^{3}\right) \frac{\partial b}{\partial \delta}\right)
$$

It is worth noting that, if bending effects are neglected, equation (13) reduces to (11) for $b_{1}=0$, i.e. for an impact directly located on the free edge.

For super-element 3, the rupture is also assumed to occur by tearing along the two horizontal supported edges. To evaluate the resistance in this case, it is possible to use Wierzbicki's formula [12], as it will be mentioned later for SE6 (see equation (21) in the related section). The force remains therefore constant, until the tearing length is sufficient enough for allowing the ship to pass through the structure. In this last situation, the crushing force is set to zero.

\subsection{Numerical validation}

For super-elements 2 and 3, the finite element models of the striking ship and struck plate are similar to those described in Section 4.2 for SE1. The data used here for LS-DYNA numerical calculations are listed in Table 6 (see Appendix 2.2). The crushing force vs. penetration curves, compared on Fig. 19, show a quite good agreement between the finite elements and analytical results. Nevertheless, the 


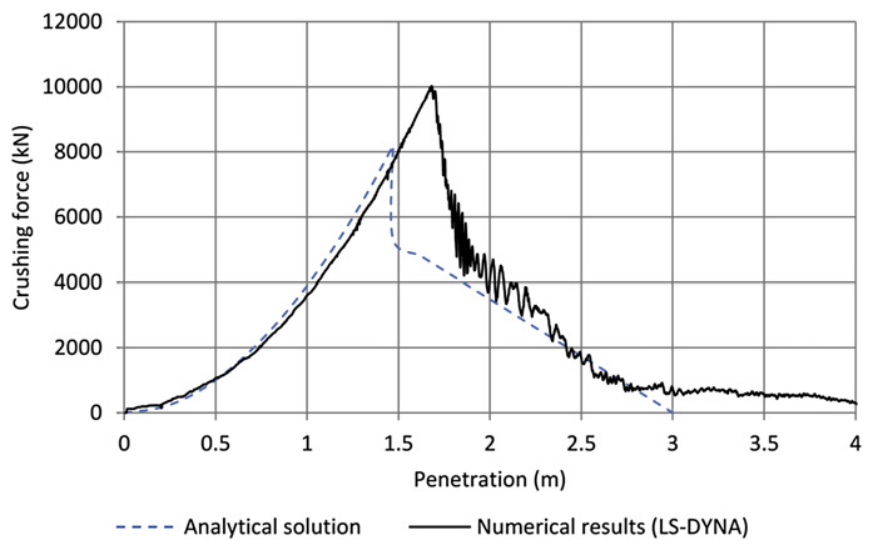

Fig. 19. Comparison between numerical and analytical solution for SE2.

rupture criteria $\psi=10 \%$ leads to a quite conservative theoretical solution, as the peak resistance predicted by the present model is lower than the one obtained using LS-DYNA.

For SE3, all data required for numerical simulations are summarized in Table 7 (see Appendix 2.2). A comparison between the analytically predicted resistance curve and the LS-DYNA numerical results is shown on Fig. 20. Using $\psi=10 \%$, the theoretical failure arises for $\delta=1.7 \mathrm{~m}$, which is more or less consistent with the numerical prediction. After rupture, the crushing resistance given by Wierzbicki's formula is also a satisfactory approximation.

\section{Description of super-element 4}

\subsection{Analytical model}

The fourth super-element (SE4) is different from the previous one, in the sense that it is not a plated structure (see Fig. 21). This time, we consider a beam submitted to a non-symmetrical impact, occurring with a certain angle. The beam is supposed to have a T-cross section and is assumed to be clamped at both extremities. This super-element corresponds typically to longitudinal or vertical stiffeners (designated by number "3" in Fig. 8). Such components are principally used for reinforcing large side panels, similar to those encountered in Section 4. In the present methodology, we have

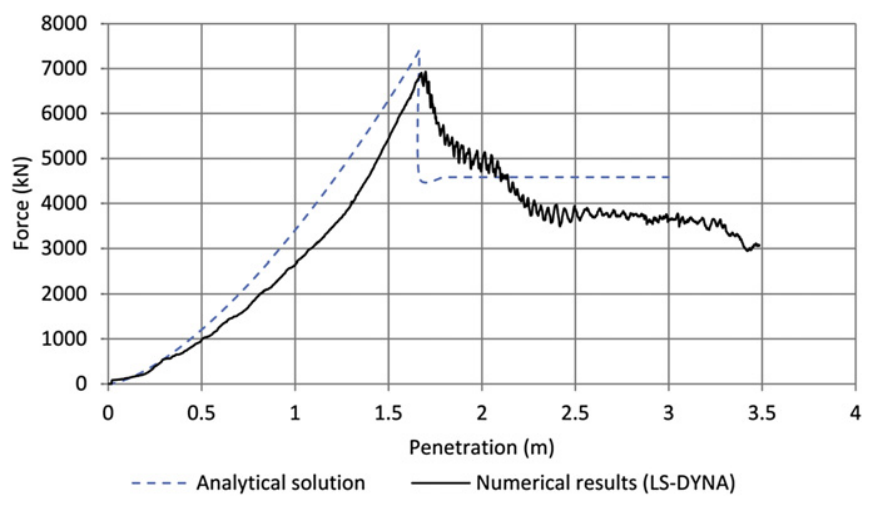

Fig. 20. Comparison between numerical and analytical solution for SE3. 


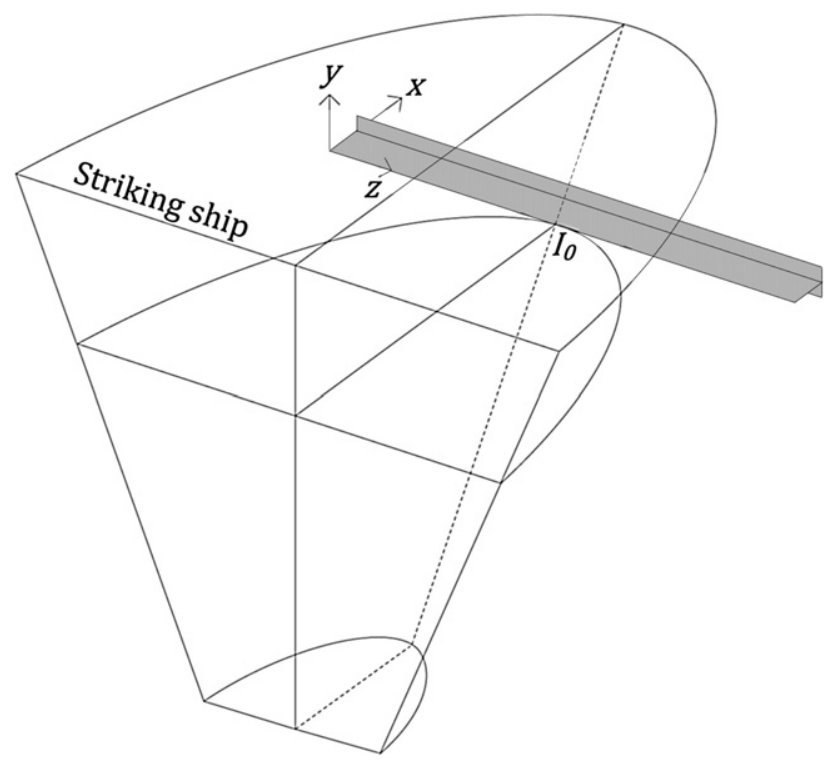

Fig. 21. General representation of the collision scenario for super-element 4.

decided to treat separately stiffeners and side panels by developing two distinct super-elements. Another solution could be to smear the stiffeners over the entire panel, as proposed for example by Zhang [6].

At the beginning of the impact, the initial contact point between the bow and the struck structure is designated by $I_{0}$. This allows for the definition of distances $a_{1}$ and $a_{2}$. As the ship goes forward, for a given penetration $\delta$, the impact point is no longer situated in $I_{0}$ but rather in $I$. The impact point $I_{0}$ is initially located at a distance $a_{1}$ from the left support (see Fig. 22a; for clarity the beam is represented by its centerline $\mathrm{AB}$ on this figure), but this length is growing as the striking ship is moving forward. In

a

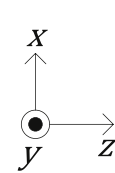

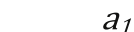

\section{t}
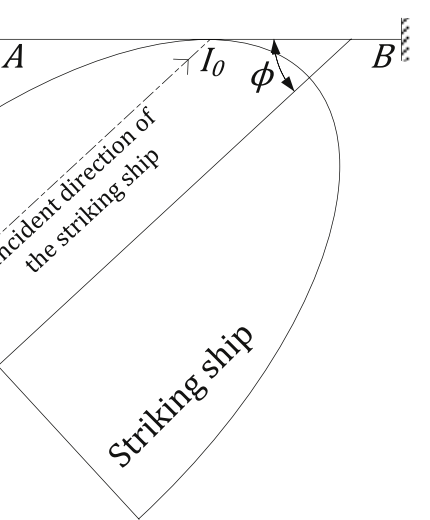

b

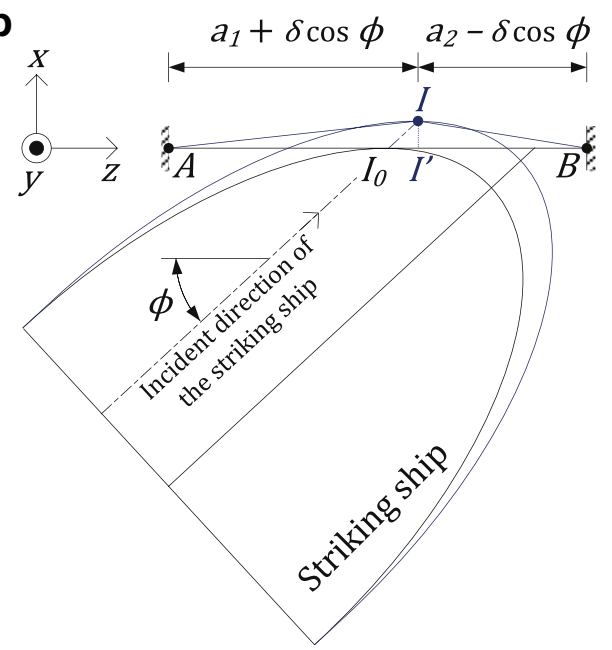

Fig. 22. (a) Initial dispositions at the beginning of the collision; (b) Deformed configuration. 
fact, for a given penetration $\delta$, the actual contact point is I (see Fig. 22b) so the horizontal distances from left and right support become:

$$
a_{1}+\delta \cos \phi \quad a_{2}-\delta \sin \phi \quad \text { with } \delta=\overline{I_{0} I}
$$

where $\phi$ is the impact angle (Fig. 22). This situation is completely similar to the case of a perpendicular impact, with a contact point location initially given by $I^{\prime}$ instead of $I$. The total penetration of the striking ship is not $\delta$ anymore, but $\delta \sin \phi$ (see Fig. 22b).

The impacted structure is assumed to withstand by formation of a mechanism involving three hinges in $A, B$ and $I$, as shown on Fig. 22b and on Fig. 23. During the plastic collapse, the structure is submitted to both flexural and extensional effects. In sections $A, B$ and $I$ where the hinges are formed, the combination between bending moment $M$ and normal force $N$ is such that the yield condition is verified. Knowing the sectional properties of super-element 4, it is possible to establish the equation of the yield locus, which provides a first additional relation between the normal force $N$ and the bending moment $M$ applied on a section where plasticity is fully developed:

$$
M=\mathcal{L}(N)
$$

where $\mathcal{L}$ is the mathematical expression of the yield locus. More details on the present assumed yield locus are summarized in Appendix 3.1.

In order to evaluate the resistance $F$ of the plastic mechanism depicted on Fig. 23, we can apply the virtual work principle. For a penetration $\delta$, the external and internal work rates are:

$$
\dot{E}_{e x t}=F \dot{\delta} \sin \phi \quad \dot{E}_{i n t}=2 M\left(\dot{\theta}_{1}+\dot{\theta}_{2}\right)+N\left(\dot{\Delta}_{1}+\dot{\Delta}_{2}\right)
$$

where

$\theta_{1}$ and $\theta_{2}$ are the rotations defined on Fig. 23

$\Delta_{1}$ and $\Delta_{2}$ are the axial extensions of segments $A I^{\prime}$ and $B I^{\prime}$

These parameters may easily be obtained by some geometrical considerations based on Fig. 23 (the corresponding mathematical expressions are summarized by equation (38) in Appendix 3.1). By equating $\dot{E}_{\text {ext }}$ and $\dot{E}_{\text {int }}$ and introducing (15) in (16), we get the following equation:

$$
F \dot{\delta} \sin \phi=2\left(\dot{\theta}_{1}+\dot{\theta}_{2}\right) \mathcal{L}(N)+N\left(\dot{\Delta}_{1}+\dot{\Delta}_{2}\right)
$$

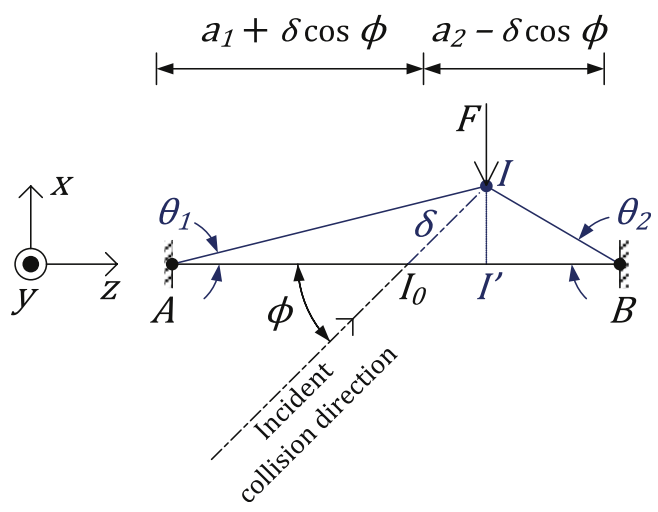

Fig. 23. Plastic mechanism for super-element 4. 
where $N$ is the only remaining unknown for evaluating $F$. This difficulty may be overcome by using a second additional link between $M$ and $N$ given by the normality condition to the yield locus. By following the procedure detailed in Appendix 3.1, we finally get the two following expressions for $F$, according to the magnitude of $N$ :

$$
\begin{array}{ll}
F=2 M_{0} \cdot\left(a_{1} a_{2}+\delta^{2} \cos ^{2} \phi\right) & \text { if } N<N_{0} \\
F=N_{0}\left(a_{1}+a_{2}\right) \cdot \delta \sin \phi \cdot \frac{a_{1} a_{2}+\delta^{2} \cos ^{2} \phi-\delta \cos \phi\left(a_{1}-a_{2}\right) / 2}{\left(a_{1}+\delta \cos \phi\right)^{2}\left(a_{2}-\delta \cos \phi\right)^{2}} & \text { if } N=N_{0}
\end{array}
$$

where $M_{0}$ and $N_{0}$ are respectively the bending and axial capacities of the T cross-section. Of course, the previous formulas remain applicable as long as there is no rupture of the beam. This is supposed to happen when the deformation reaches a critical value $\psi$. Then, the resistance of the super-element is set to zero.

\subsection{Numerical validation}

In order to validate the analytical developments of Section 6.1, some numerical simulations have been performed using LS-DYNA. The striking bow is the same as the one used previously. The impacted stiffener is modeled by 160 Hughes-Liu beam elements. The dimensions of the cross-section and the description of the collision scenario are given in Appendix 3.2.

As shown on Fig. 24, there is a quite good agreement between numerical and analytical results. Rupture occurs for a perpendicular penetration more or less equal to $2.5 \mathrm{~m}$. The mathematical model gives a slightly different value, but the difference with the one furnished by LS-DYNA is still acceptable.

\section{Description of super-element 6}

\subsection{Analytical model}

This new super-element is a plate, simply supported on three edges and remaining free on the last one. The collision scenario is depicted on Fig. 25, where it is shown that the structure is impacted at point $I_{0}$ on its free edge. Initially, the distances between $I_{0}$ and the supported edges are $a_{1}$ and $a_{2}$.

We assume that the ship is moving into the plate with a constant orientation given by the angle $\phi$. Consequently, for a given penetration $\delta$, the current contact point is located in $I$ (see Fig. 26, where, for clarity, the striking vessel is not represented but follows the direction given by $d_{1}$ ) and we have:

$$
\delta=\overline{I_{0} I}
$$

For this value of $\delta$, the corresponding deformation pattern is a fold of height $4 H$, having a left part of increasing length $a_{1}+\delta \cos \phi$ and a right part with a decreasing length $a_{2}-\delta \cos \phi$, as illustrated on Fig. 26.

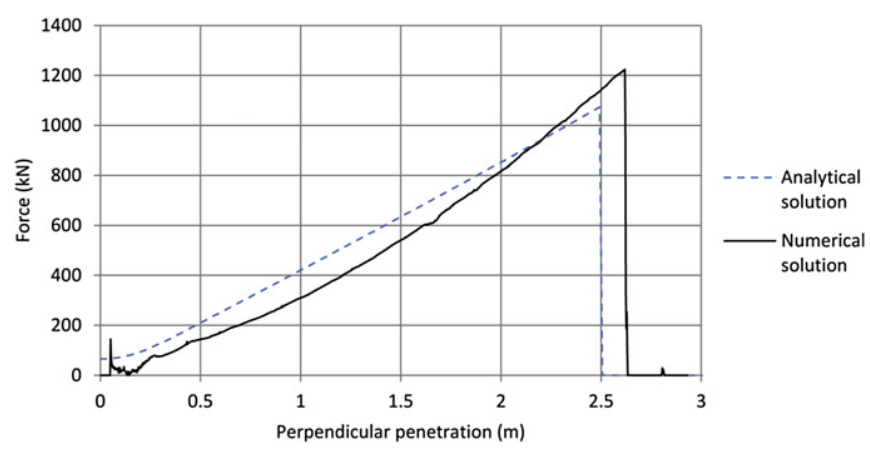

Fig. 24. Comparison between numerical and analytical solution for SE4. 


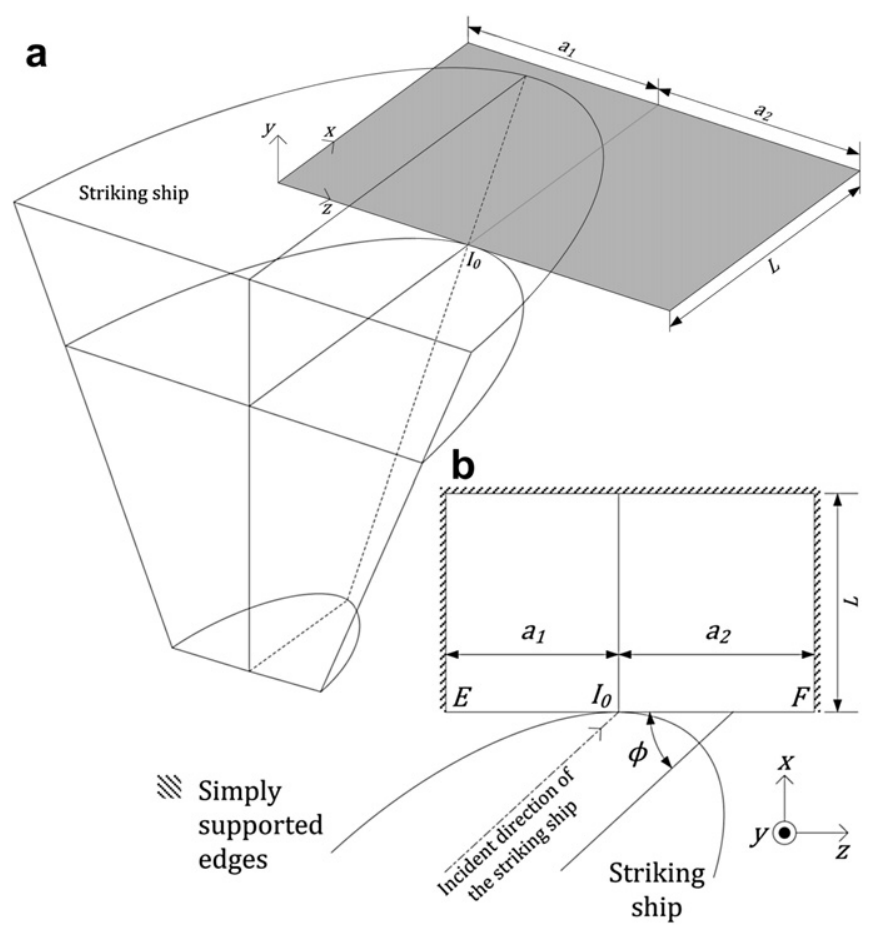

Fig. 25. General representation of the collision scenario for super-element 6 .

In order to assess the crushing resistance of this super-element, we have to evaluate the internal energy dissipated during the crushing process, and it is therefore required to define a compatible displacement field. This may be done in accordance with Fig. 27, where it is shown that the plastic mechanism implies two different effects:

- Bending effects: one fold involves three triangular surfaces EAB, EBC and ECD (see Fig. 27b), which are assumed to rotate around four plastic hinges located in EA, EB, EC and ED. As a result, a part $E_{\mathrm{b}}$ of the dissipated internal energy is produced by the rotations $\theta$ (see Fig. 26) confined in these lines.

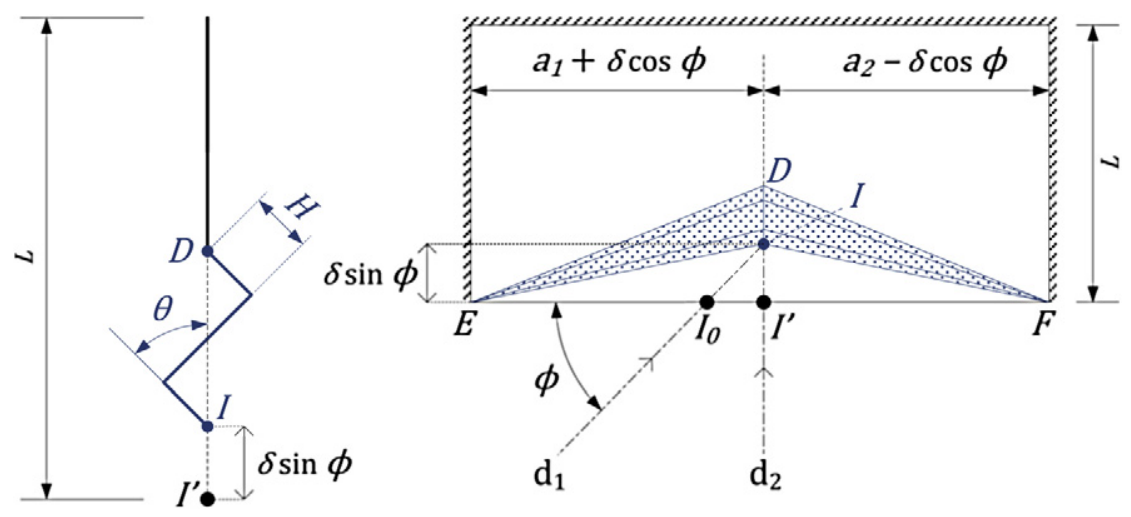

Fig. 26. Deformed configuration of super-element 6 . 
a

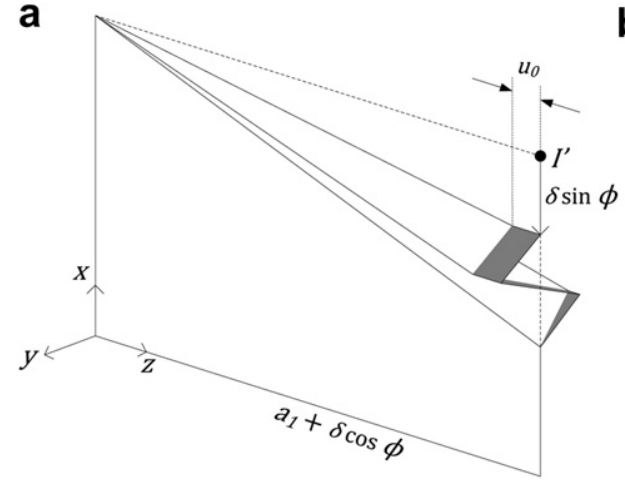

b

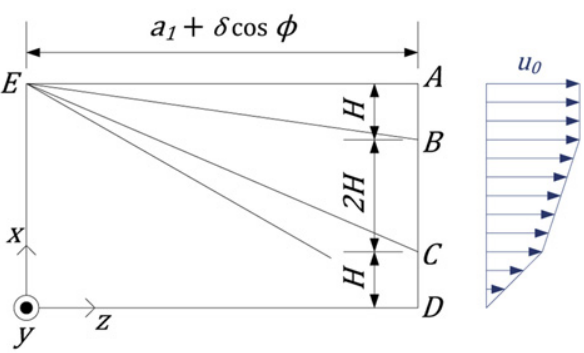

Fig. 27. (a) Folding mechanism; (b) Displacement field.

- Membrane effects: if the above-mentioned triangular surfaces were moving rigidly around the plastic hinges, it would impossible to maintain the compatibility along line ID on Fig. 26. Consequently, the material has to be strained along $z$-direction (see the gray zone on Fig. 27a) in order to restore the contact between the two part EID and FID of the fold. This straining may be achieved by imposing a displacement field $u(x, z)$ oriented along $z$-direction (see Fig. 27b) and leading to a membrane energy dissipation $E_{\mathrm{m}}$.

The rotation angle $\theta$ (see Fig. 26) and the maximum displacement $u_{0}$ are the two fundamental parameters defining the mentioned plastic mechanism. They allow for the derivation of the crushing resistance of one fold, as exposed in Appendix 4.1.

However, when $\delta=4 H$, the current fold shown on Fig. 26 is completely closed and the previous plastic mechanism has to be extended for subsequent values of the penetration. To do so, we can assume that the folding process described here over is repeated as long as $\delta \leq L$, i.e. as long as the superelement is not completely crushed. By following a similar procedure than [21], we finally obtained the following resistance of super-element 6 , when $N_{\mathrm{f}}-1$ folds are already completely crushed:

$$
F=\frac{3 \pi}{16 H} \sigma_{0} t_{p}^{2}\left(a_{1}+a_{2}\right)+\frac{11}{8} \sigma_{0} t_{p} H \sum_{i=1}^{N_{f}}(\delta \sin \phi-4(i-1) H) \cdot F_{i}(\delta)
$$

where:

$$
\begin{aligned}
& F_{i}(\delta)=\frac{2 a_{1}+\delta \cos \phi+4(i-1) H \cot \phi}{\left(a_{1}+\delta \cos \phi\right)^{2}}+\frac{2 a_{2}-\delta \cos \phi-4(i-1) H}{\left(a_{2}-\delta \cos \phi\right)^{2}} \\
& N_{f}=\left[\frac{\delta \sin \phi}{4 H}\right]
\end{aligned}
$$

Of course, the present super-element has a limited level of resistance because the plate ruptures before it has been crushed over its entire length $L$. Most of the time, this happens by tearing along the supported edges as illustrated by Fig. 28, which leads to a subsequent particular mechanism called "concertina tearing". In order to model this particular behavior, we can suppose that the switch between the folding mechanism (Fig. 27) and the concertina tearing (Fig. 28) takes place when the maximal deformation of the super-element reaches a critical value $\psi$. As soon as this criterion is satisfied, the crushing resistance is not set immediately to zero. In fact, during the concertina tearing 


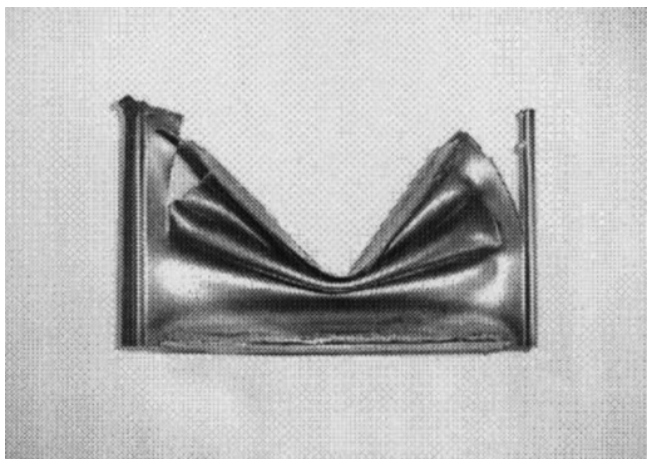

Fig. 28. Concertina tearing - the figure is extracted from [21].

mechanism, the plate keeps a certain resistance level, which was already studied by Wierzbicki [12]. According to [12], the crushing force after rupture is constant and given by:

$$
F=4.33 \sigma_{0} t_{p}^{5 / 3}\left(a_{1}+a_{2}\right)^{1 / 3}+\frac{8}{3} R_{m} t_{p}
$$

where $R_{\mathrm{m}}$ is the tearing resistance of steel. Of course, formula (21) remains valid as long as the element is not completely crushed over its total length $L$.

\subsection{Numerical validation}

In order to validate the model exposed previously, some numerical simulations have been performed by using LS-DYNA. The values of all the collision parameters are listed in Appendix 4.2. The material law is still the same as for super-element 1 (see Table 5 and Fig. 35).

In order to set clearly the impact scenario, Fig. 29 shows the finite elements models of both the striking ship and the struck structure (for clarity, the scale between the bow and the plate is not respected), on which we have also indicated the reference frame $(x, y, z)$ and the collision angle $\phi$. The collided edge has a total breadth of $8 \mathrm{~m}$, while the depth of the element is $12 \mathrm{~m}$. Of course, for current ship architecture, it is quite seldom to encounter non-stiffened plates exhibiting such dimensions.

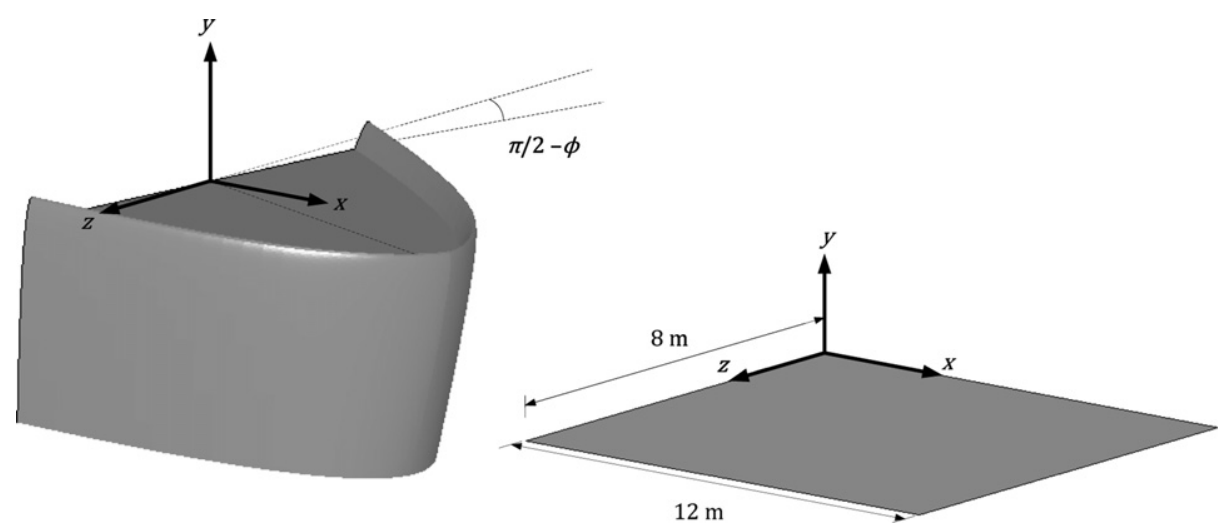

Fig. 29. Finite element modeling of the striking ship and the impacted plate. 
Nevertheless, it is important to recall that the stiffeners present on such panels are modeled as SE5, as they are considered to be crushed axially during the impact.

For the present simulation, the plate is simply supported on three edges and is totally free on the impacted one. It is modeled with 38,400 Belytschko-Tsay shell elements [27], which corresponds to a mesh size of $5 \mathrm{~cm} \times 5 \mathrm{~cm}$. The striking bow is the same as for super-element 1 .

Here again, a comparison with LS-DYNA resulting curve shows a quite good agreement (see Fig. 30). The numerical curve shows a peak resistance for a penetration of $2.5 \mathrm{~m}$, for which rupture occurs. After that, the resistance decreases and stabilization arises for a crushing force of about $6000 \mathrm{kN}$, corresponding more or less to the resistance predicted by equation (21) for the concertina tearing mode. This behavior is well-captured by the analytical curve, even if the theoretical rupture occurs a bit later, for a penetration of $2.7 \mathrm{~m}$. This is due to the choice $\psi=10 \%$, which is may be optimistic for super-element 6 .

\section{Validation on realistic collision scenarios}

\subsection{Comparison with finite element results}

The super-elements presented in previous sections have been implemented in a global calculation program named SHARP, which couples internal and external mechanics [19]. In order to validate the developed formulations on realistic oblique collision scenarios, several impact simulations have been performed for different collision angles and resulting penetrations. Internal energies have been compared with those obtained using nonlinear LS-DYNA/MCOL finite element calculations [27]. One of these simulations is illustrated in Fig. 31, where a 350,000 DWT FPSO side is struck by a 140,000 DWT crude oil carrier at a velocity of 1.5 knots. The striking ship is assumed to be rigid. The FPSO is at rest and is collided between two transverse frames. The main characteristic of both ships are listed in Table 2 .

In finite element simulations, rupture of FPSO side shell is modeled using erosive plastic behavior law for steel material. When the plastic strain calculated in shell elements exceeds a threshold value of $20 \%$, their crushing resistance is set to zero. Finite elements and SHARP calculations are run for $75^{\circ}$ and $60^{\circ}$ collision angles. Time histories of resulting penetration into struck ship are compared in Fig. 32a. The difference between finite element and SHARP maximum penetration does not exceed $5 \%$. A comparison of internal energy time histories for $75^{\circ}$ impact angle case is shown on Fig. 32b.

\subsection{Sensitivity analysis to impact angle}

The sensitivity to impact angle is then studied by considering the collision between a dry cargo vessel and a passenger ship. The main characteristics of these vessels are given in Table 3 . The impact angle $\beta$ varies from $30^{\circ}$ to $150^{\circ}$ and collisions for two longitudinal impact locations are investigated. In the first configuration, the cargo vessel impacts the passenger ship on a bulkhead located near

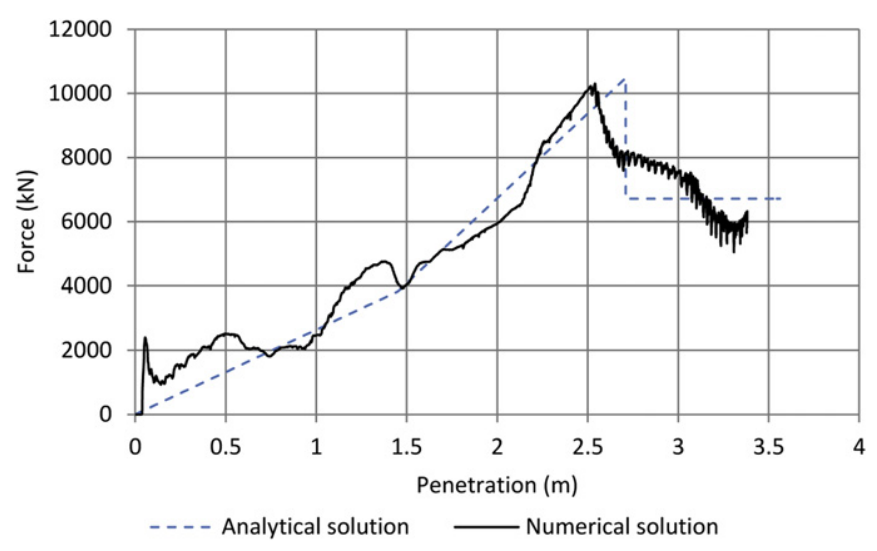

Fig. 30. Comparison between numerical and analytical solution for SE6. 

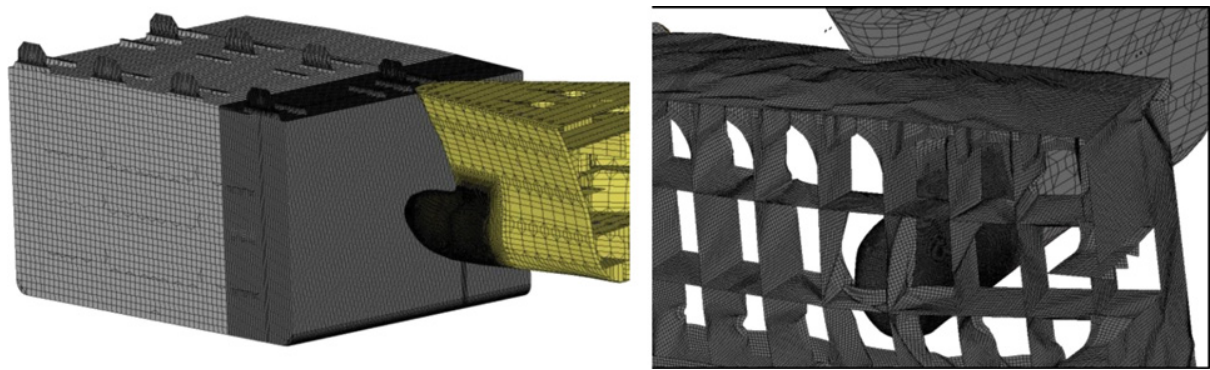

Fig. 31. View of finite element ship models.

Table 2

FPSO and crude oil carrier main characteristics.

\begin{tabular}{lll}
\hline & Struck FPSO & Striking crude oil carrier \\
\hline Length & $280 \mathrm{~m}$ & $200 \mathrm{~m}$ \\
Breadth & $60 \mathrm{~m}$ & $48 \mathrm{~m}$ \\
Depth & $33 \mathrm{~m}$ & $25 \mathrm{~m}$ \\
Draft & $23 \mathrm{~m}$ & $13 \mathrm{~m}$ \\
DWT & $350,000 \mathrm{t}$ & $140,000 \mathrm{t}$ \\
\hline
\end{tabular}

amidships, at $60 \mathrm{~m} /$ aft PP (Fig. 33a). In the second configuration, the passenger ship is struck between the aft and the first transversal bulkheads, at $10 \mathrm{~m} / \mathrm{aft}$ PP. For both configurations, the cargo vessel is assumed to be rigid, the impact velocity is equal to 7 knots and the passenger ship is initially at rest.

The struck ship damage penetration is plotted on Fig. 33b as a function of the impact angle. The first impact point being located near the struck ship center of gravity, the obtained curve is symmetric around $90^{\circ}$, as expected. The maximum indentation is observed for $65^{\circ}$ and $115^{\circ}$ impact angles. On the other hand, when collision occurs near the aft bulkhead, the passenger ship sway movement is more important and the ship "escapes" from striking bow for impact angles higher than $130^{\circ}$, which leads of course to lower indentation.

Extend of longitudinal damage is plotted in Fig. 34 as a function of impact angle for the first impact longitudinal location, i.e. near amidships. It's observed that damage lengths are largest for collision angles of approximately $60^{\circ}$ and $120^{\circ}$. A.J. Brown reported in [31] such damage length calculations and obtained similar results, even if the dissymmetry of the curves presented by Brown may be explicated by the fact that the different studied struck ships had non zero surge velocity.
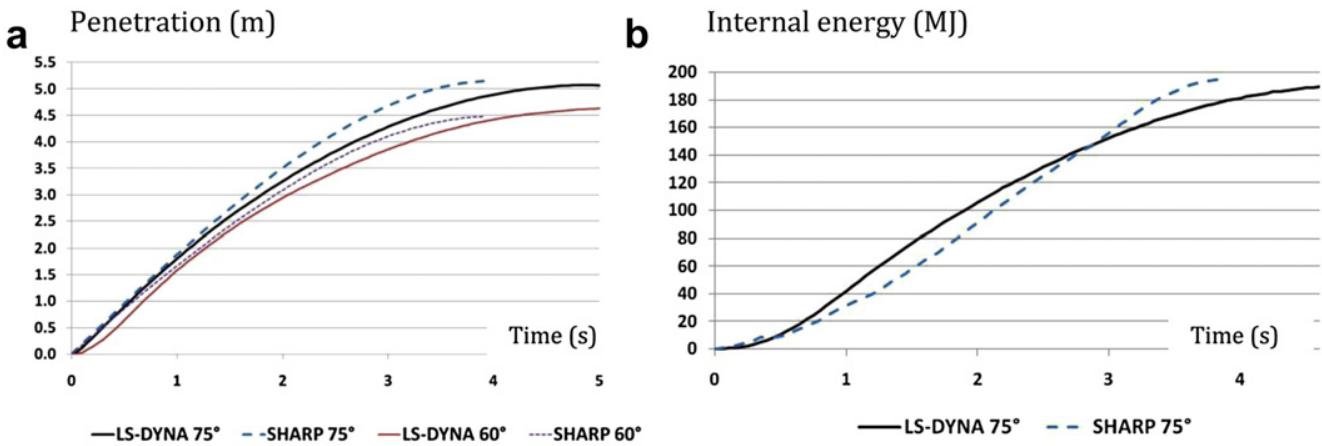

Fig. 32. Penetration and internal energies: SHARP compared to LS-DYNA results. 
Table 3

Dry cargo vessel and passenger ship main characteristics.

\begin{tabular}{lll}
\hline & Striking cargo & Struck passenger ship \\
\hline Length & $168 \mathrm{~m}$ & $150 \mathrm{~m}$ \\
Breadth & $20 \mathrm{~m}$ & $20 \mathrm{~m}$ \\
Depth & $15 \mathrm{~m}$ & $13 \mathrm{~m}$ \\
Draft & $6.8 \mathrm{~m}$ & $5 \mathrm{~m}$ \\
DWT & $6300 \mathrm{t}$ & $6000 \mathrm{t}$ \\
\hline
\end{tabular}

a View of collision scenario (1 ${ }^{\text {st }}$ config. $)$

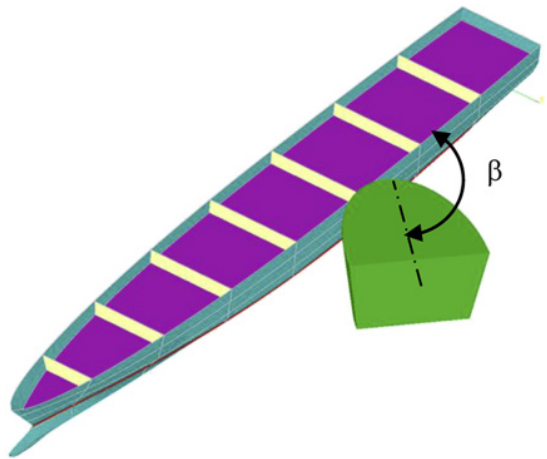

b Penetration $(\mathrm{m})$

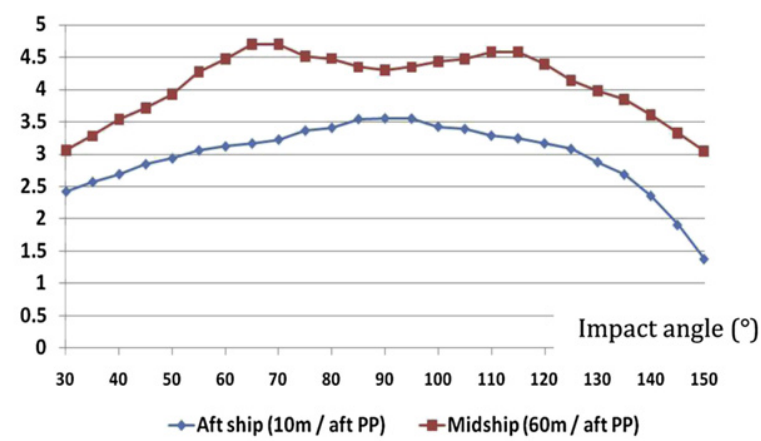

Fig. 33. Sensitivity of damage penetration to impact angle.

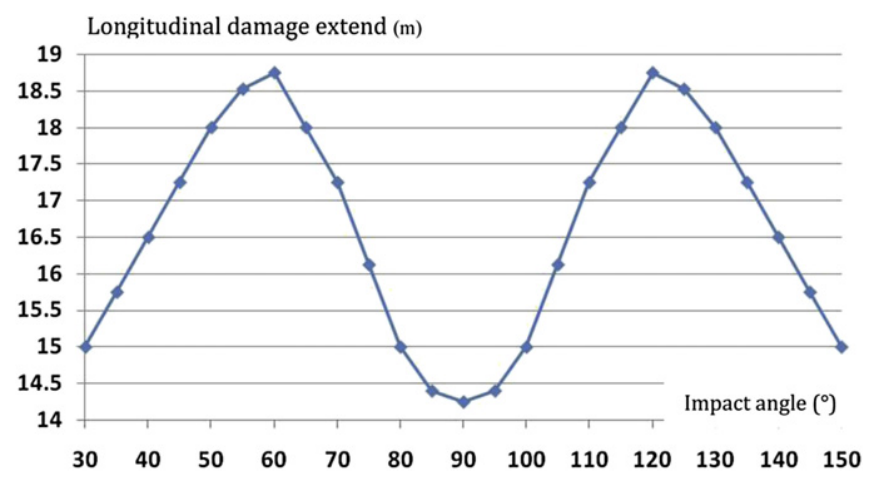

Fig. 34. Sensitivity of longitudinal damage extend to impact angle.

\section{Conclusion}

Through this article, a kind of "updating" of the super-element method was exposed. In the first part of the text, the whole method was shortly recalled and some explanations were given on the way to deal with the internal and external mechanics. The second part of the article was principally devoted to the description of analytical procedures, which were used to extend the super-elements method to the case of oblique collisions.

The first step for analyzing non-perpendicular collisions between ships was the use of the virtual work principle. By combining this theorem with a rigid-plastic material law, a general analytical formulation has been derived in order to simply evaluate the energy rate related to the plastic collapse of various super-elements. 
Therefore, to completely treat oblique collisions cases, six different super-elements were developed. For each of them, the assumed deformation mechanisms were described throughout the paper. With these rather basic mechanisms, it was possible to evaluate the relation between the resistance force and the penetration of the striking ship into the struck ship. These analytical formulations were validated by comparison with numerical simulations performed by using the finite elements code LSDYNA. For each of the described super-elements, it was established that analytical and numerical results were in quite good accordance.

The closed-form expressions developed to evaluate the crushing force of these super-elements were then implemented in a global calculation program, named SHARP, which couples internal and external mechanics. In order to validate the new program version on realistic oblique impact scenarios, the collision between a crude cargo liner and a FPSO was modeled using a finite element code and the SHARP program. Several impact simulations were performed for different collision angles. Penetrations and internal energies calculated by SHARP program were then compared with those obtained using the nonlinear FEM code LS-DYNA.

Finally, by modeling the collision between a dry cargo vessel and a passenger ship, such a rapid prediction tool was advantageously used to study the sensitivity of damage to impact angle. Penetrations and longitudinal damage length curves obtained using SHARP program were found to be similar to those presented by A.J. Brown in [31].

\section{Acknowledgments}

This work has been performed in the frame of the joint industry project "SHARP2" (Ship Hazardous Aggression Research Program). The aim of SHARP2 is to deliver a dedicated software and associated guideline for marine structure for resisting to aggression damage: ship collision and collision against icebergs. ICAM and Principia RD would like to thank DCNS and Bureau Veritas for their technical engagement in defining the scope of work and their financial support to SHARP2 project.

\section{Appendix 1. Details on SE1 development and validation}

In this section, we summarize some additional mathematical results and present the required data useful for numerical validations with LS-DYNA. Additional information is also given on material modeling.

\section{Appendix 1.1. Analytical developments}

The evaluation of the strain rates tensor is realized in accordance with Green's definitions. For surface I, we have:

$$
\begin{aligned}
& \varepsilon_{z z}=\frac{1}{2}\left(\frac{\partial w}{\partial z}\right)^{2} \\
& \rightarrow \dot{\varepsilon}_{z z}=\frac{\partial w}{\partial z} \frac{\partial \dot{w}}{\partial z}=\delta \dot{\delta} \sin ^{2} \phi\left(\frac{y}{b}\right)^{2 n} \frac{n^{2} z^{2 n-2}}{\left(a_{1}+\delta \cos \phi\right)^{2 n}} \frac{a_{1}+(1-n) \delta \cos \phi}{a_{1}+\delta \cos \phi} \\
& \varepsilon_{y y}=\frac{1}{2}\left(\frac{\partial w}{\partial y}\right)^{2} \\
& \rightarrow \dot{\varepsilon}_{y y}=\frac{\partial w}{\partial y} \frac{\partial \dot{w}}{\partial y}=\delta \dot{\delta} \sin ^{2} \phi\left(\frac{z}{a_{1}+\delta \cos \phi}\right)^{2 n} \frac{n^{2} y^{2 n-2}}{b^{2 n}} \frac{a_{1}+(1-n) \delta \cos \phi}{a_{1}+\delta \cos \phi}
\end{aligned}
$$




$$
\begin{aligned}
& \varepsilon_{z y}=\frac{1}{2}\left(\frac{\partial w}{\partial z} \frac{\partial w}{\partial y}\right) \\
& \rightarrow \dot{\varepsilon}_{z y}=\frac{1}{2}\left(\frac{\partial w}{\partial z} \frac{\partial \dot{w}}{\partial y}+\frac{\partial \dot{w}}{\partial z} \frac{\partial w}{\partial y}\right)=\delta \dot{\delta} \sin ^{2} \phi \frac{n z^{2 n-1}}{\left(a_{1}+\delta \cos \phi\right)^{2 n}} \frac{n y^{2 n-1}}{b^{2 n}} \frac{a_{1}+(1-n) \delta \cos \phi}{a_{1}+\delta \cos \phi}
\end{aligned}
$$

The three previous expressions are inserted into equation (4) for deriving the membrane energy rate $\dot{E}_{I}$ associated with surface I. If we achieve similar developments for the three remaining surfaces, after calculation, we get:

$$
\begin{aligned}
& \dot{E}_{I}=\frac{2 \sigma_{0} t_{p}}{\sqrt{3}} \frac{n^{2}}{4 n^{2}-1}\left(\frac{1}{\left(a_{1}+\delta \cos \phi\right)^{2}}+\frac{1}{b_{1}^{2}}\right) b_{1}\left(a_{1}+(1-n) \delta \cos \phi\right) \delta \dot{\delta} \sin ^{2} \phi \\
& \dot{E}_{I I}=\frac{2 \sigma_{0} t_{p}}{\sqrt{3}} \frac{n^{2}}{4 n^{2}-1}\left(\frac{1}{\left(a_{1}+\delta \cos \phi\right)^{2}}+\frac{1}{b_{2}^{2}}\right) b_{2}\left(a_{1}+(1-n) \delta \cos \phi\right) \delta \dot{\delta} \sin ^{2} \phi \\
& \dot{E}_{I I I}=\frac{2 \sigma_{0} t_{p}}{\sqrt{3}} \frac{n^{2}}{4 n^{2}-1}\left(\frac{1}{\left(a_{2}-\delta \cos \phi\right)^{2}}+\frac{1}{b_{1}^{2}}\right) b_{1}\left(a_{2}-(1-n) \delta \cos \phi\right) \delta \dot{\delta} \sin ^{2} \phi \\
& \dot{E}_{I V}=\frac{2 \sigma_{0} t_{p}}{\sqrt{3}} \frac{n^{2}}{4 n^{2}-1}\left(\frac{1}{\left(a_{2}-\delta \cos \phi\right)^{2}}+\frac{1}{b_{1}^{2}}\right) b_{1}\left(a_{2}-(1-n) \delta \cos \phi\right) \delta \delta \dot{\delta} \sin ^{2} \phi
\end{aligned}
$$

Finally, the total membrane energy rate $\dot{E}_{m}$ dissipated by super-element 1 is simply obtained by adding the four individual contributions of surfaces I-IV:

$$
\dot{E}_{m}=\dot{E}_{I}+\dot{E}_{I I}+\dot{E}_{I I I}+\dot{E}_{I V}
$$

\section{Appendix 1.2. Collision scenario}

In order to validate the formulas detailed here above, they have been compared with results given by LS-DYNA. The numerical values defining the collision scenario are listed in Table 4.

\section{Table 4}

Collision parameters for super-element 1.

\begin{tabular}{lll}
\hline Horizontal length of the plate & $a_{1}+a_{2}$ & $8 \mathrm{~m}$ \\
Vertical length of the plate & $b_{1}+b_{2}$ & $6 \mathrm{~m}$ \\
Horizontal initial position of contact point $I_{0}$ & $a_{1}$ & $4 \mathrm{~m}$ \\
& $a_{2}$ & $4 \mathrm{~m}$ \\
Vertical initial position of contact point $I_{0}$ & $b_{1}$ & $3 \mathrm{~m}$ \\
& $b_{2}$ & $3 \mathrm{~m}$ \\
Plate thickness & $t_{\mathrm{p}}$ & $2.5 \mathrm{~cm}$ \\
Speed of the striking ship & $V_{1}$ & $2 \mathrm{~m} / \mathrm{s}$ \\
Mass of the striking ship & $M_{1}$ & 1426 ton \\
Collision angle & $\phi$ & $45^{\circ}$ \\
Friction coefficient & $\mu$ & 0.3 \\
\hline
\end{tabular}




\section{Appendix 1.3. Material law}

For numerical simulations using LS-DYNA, we assume that the behavior of steel may be satisfactorily represented by a bilinear stress-strain relation. In this case, the elastic-plastic stress-strain curve is split into two distinct portions (see Fig. 35). The first part of the curve corresponds to the elastic phase. The curve is linear, with an inclination corresponding to Young's Modulus $E_{\mathrm{Y}}$. When the yield stress $\sigma_{0}$ is reached, the plastic phase begins. The relation is still linear, but the slope has changed and is given by the tangent modulus $E_{\mathrm{T}}$. In the present model, the strain-rate effect is not taken into account. The values of the different parameters are listed in Table 5.

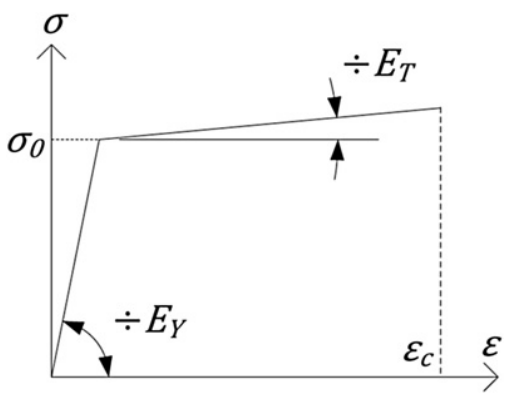

Fig. 35. stress-strain relation of steel.

Table 5

Steel material characteristics.

\begin{tabular}{lll}
\hline Property & Notation & Value \\
\hline Density & $\rho$ & $7850 \mathrm{~kg} / \mathrm{m}^{3}$ \\
Poisson's ratio & $\nu$ & 0.33 \\
Yield stress & $\sigma_{0}$ & $240 \mathrm{MPa}$ \\
Young's modulus & $E_{\mathrm{Y}}$ & $210,000 \mathrm{MPa}$ \\
Tangent modulus & $E_{\mathrm{T}}$ & $1018 \mathrm{MPa}$ \\
Critical strain & $\varepsilon_{\mathrm{c}}$ & $25 \%$ \\
\hline
\end{tabular}

\section{Appendix 2. Details on SE2 and SE3 developments and validations} DYNA.

In this section, we present some mathematical results and the data used for validations with LS-

\section{Appendix 2.1. Analytical developments}

For SE2 and SE3 super-elements derivation, we assume that the bow of the striking ship has an elliptical shape. The ellipse is described by curve $T$ on Fig. 36. Its center is designated by $Q$ and is the origin of a local coordinate system $\left(x_{\mathrm{s}}, y_{\mathrm{s}}, z_{\mathrm{s}}\right)$ moving with the ship. In this coordinate system, the equation of $\Gamma$ is:

$$
\Gamma \equiv \frac{x_{s}^{2}}{r^{2}}+\frac{z_{s}^{2}}{q^{2}}=1
$$

where $r$ and $q$ are the elliptical radii (see Fig. 36). In fact, this formula is only valid at the beginning of the impact, when the ship has just entered in contact with the plate at point $I_{0}$. However, when it is moving forward, equation (30) has to be updated to account for the penetration $\delta$. As we assume that the bow is following the same direction than $z_{\mathrm{s}}$, we have: 


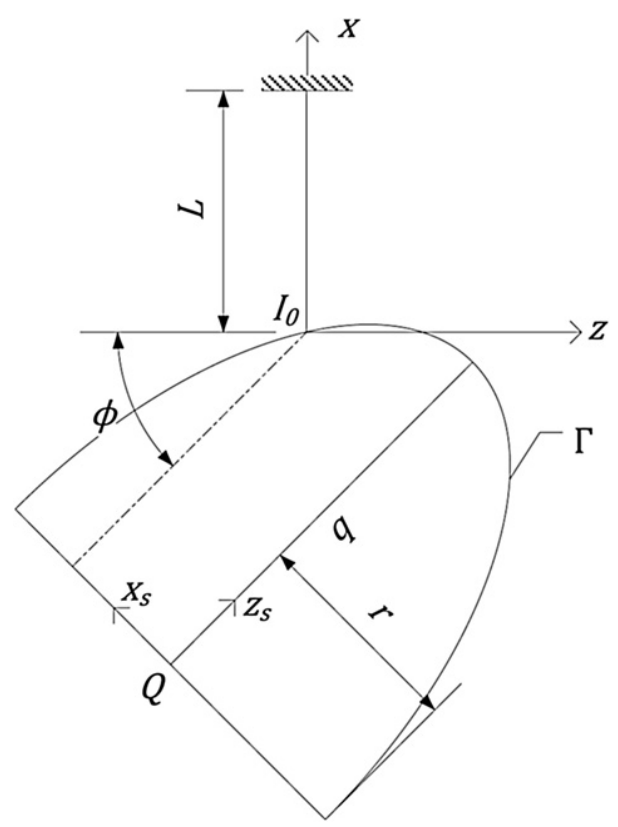

Fig. 36. Parameterization of the bow of the striking ship.

$$
\Gamma \equiv \frac{x_{s}^{2}}{r^{2}}+\frac{\left(z_{s}-\delta\right)^{2}}{q^{2}}=1
$$

Radii $r$ and $q$ must be defined by the user as input data. They have to be chosen for representing satisfactorily the real geometry of the bow.

As the displacement field is given in $(x, y, z)$ axes, it is more convenient to define $I$ with these coordinates. If, in this reference frame, we designate by $\left(x_{0}, y_{0}, z_{0}\right)$ the location of point $Q$ at the moment when the ship entered in contact with the plate at point $I_{0}$, we can transform equation (31) to obtain:

$$
\Gamma \equiv\left[\frac{\left(x-x_{0}\right) \cos \phi-\left(z-z_{0}\right) \sin \phi}{r}\right]^{2}+\left[\frac{\left(x-x_{0}\right) \sin \phi+\left(z-z_{0}\right) \cos \phi-\delta}{q}\right]^{2}=1
$$

Equation (32) allows for the determination of the two parameters $p$ and $b$ (for recall, they are defined by $p=\overline{I_{0} I}$ and $b=\overline{I_{0} B}$ on Fig. 14b):

- The evaluation of $b$ required in fact to position point $B$ along the $x$-axis of Fig. 14b. This can be analytically achieved by calculating the intersection of $\Gamma$ with $x$-axis.

- For evaluating $p$, it is necessary to know the location of point $I$ in the plane $(x, z)$, as shown on Fig. 16b. The closed-form expression of $p$ is then obtained by combining equation (32) and the imposed condition (8).

As the two previous calculations are quite fastidious, they have not been reported here. In this appendix, it is probably more relevant to give some additional details on the displacement field assumed for SE2. As mentioned previously (see Fig. 16b), we suppose that the plastic mechanism is formed by two triangles $\mathrm{I}_{0} \mathrm{BD}$ and $\mathrm{I}_{0} \mathrm{BG}$, which have rotated along two plastic hinges $\mathrm{BD}$ and $\mathrm{BG}$ to reach their deformed configurations IBD and IBG. We will here only focus on the upper triangle $\mathrm{I}_{0} \mathrm{BD}$, but the procedure is similar for $\mathrm{I}_{0} \mathrm{BG}$. 
During the transformation of $\mathrm{I}_{0} \mathrm{BD}$ to IBD, we assume that there is only an axial straining of the fibers parallel to $y$-axis (see Fig. 37a), combined with a rotation along line $\mathrm{BD}$, where bending effects are confined. Under the hypothesis that $p \ll a_{1}$, the transition from $\mathrm{I}_{0} \mathrm{BD}$ to IBD requires only the definition of a displacement field $v(x, y)$ oriented along $y$-axis, which is idealized as varying linearly:

$$
v(x, y)=v_{0} \frac{a_{1}-x}{a_{1}} \frac{b-y}{b}
$$

where $v_{0}$ is the vertical displacement of point $I_{0}$. By considering Fig. $37 \mathrm{~b}$, we see that for reaching its deformed configuration ID, fiber $\mathrm{I}_{0} \mathrm{D}$ has to be submitted to an axial extension given by:

$$
v_{0}=\sqrt{a_{1}^{2}+p^{2}}-a_{1} \approx \frac{p^{2}}{2 a_{1}} \quad p \ll a_{1}
$$

The combination of (33) and (34) finally leads to (9). With help of this displacement field, it is now possible to derive the total energy $E_{\mathrm{m}}$ only dissipated by membrane straining along $y$-axis. To do so, we just need to evaluate the deformation $\varepsilon_{\mathrm{yy}}$ according to Green's formula, as mentioned previously. Finally, the last two parameters to establish for the complete definition of the crushing force $F$ are the rotation rate $\partial \theta_{1} / \partial \delta$ and $\partial \theta_{2} / \partial \delta$ in equation (11). By using (10), for the plastic hinge BD for example, we have:

$$
\frac{\partial \theta_{1}}{\partial \delta}=\frac{a_{1}}{\sqrt{4 b^{2}\left(b^{4}+a^{2} p^{2}\right)-a^{2} p^{4}}}\left[b \frac{2 b^{2}-p^{2}}{a_{1}^{2}+b^{2}} \frac{\partial b}{\partial \delta}+2 p\left(\frac{\partial p}{\partial \delta}-\frac{p}{b} \frac{\partial b}{\partial \delta}\right)\right]
$$
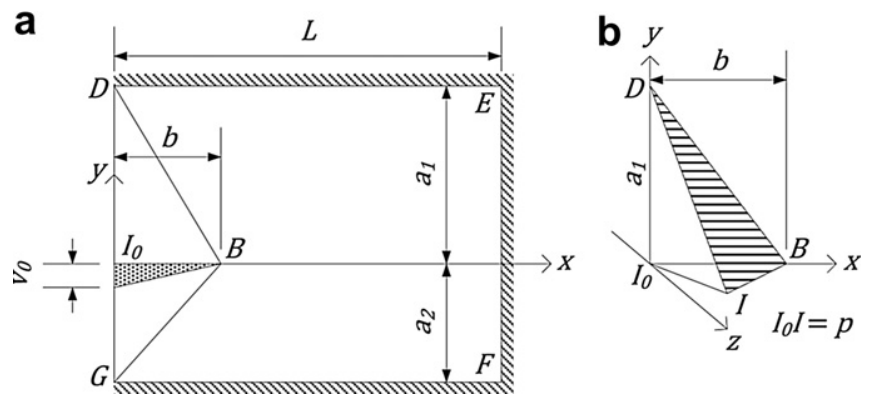

Fig. 37. Displacement field assumed for SE2.

\section{Appendix 2.2. Collision scenario}

The numerical values defining the collision scenario are listed in Table 6 for super-element 2 and in Table 7 for super-element 3 .

\section{Table 6}

Collision parameters for super-element 2 .

\begin{tabular}{lll} 
Vertical length of the plate & $a_{1}+a_{2}$ & $8 \mathrm{~m}$ \\
Horizontal length of the plate & $L$ & $6 \mathrm{~m}$ \\
Vertical initial position of contact point $I_{0}$ & $a_{1}$ & $4 \mathrm{~m}$ \\
& $a_{2}$ & $4 \mathrm{~m}$ \\
Plate thickness & $t_{\mathrm{p}}$ & $2.5 \mathrm{~cm}$ \\
Speed of the striking ship & $V_{1}$ & $5 \mathrm{~m} / \mathrm{s}$ \\
Mass of the striking ship & $M_{1}$ & 1426 ton \\
Collision angle & $\phi$ & $45^{\circ}$ \\
\hline
\end{tabular}


Table 7

Collision parameters for super-element 3.

\begin{tabular}{lll}
\hline Horizontal length of the plate & $a_{1}+a_{2}$ & $8 \mathrm{~m}$ \\
Vertical length of the plate & $b_{1}+b_{2}$ & $8 \mathrm{~m}$ \\
Vertical initial position of contact point $I_{0}$ & $a_{1}$ & $4 \mathrm{~m}$ \\
& $a_{2}$ & $4 \mathrm{~m}$ \\
Horizontal initial position of contact point $I_{0}$ & $b_{1}$ & $3 \mathrm{~m}$ \\
& $b_{2}$ & $5 \mathrm{~m}$ \\
Plate thickness & $t_{\mathrm{p}}$ & $5 \mathrm{~cm}$ \\
Speed of the striking ship & $V_{1}$ & 1426 ton \\
Mass of the striking ship & $M_{1}$ & $75^{\circ}$ \\
Collision angle & $\phi$ & \\
\hline
\end{tabular}

\section{Appendix 3. Details on SE4 development and validation}

In this section, we summarize some additional mathematical results and present the required data useful for validations with LS-DYNA.

\section{Appendix 3.1. Analytical developments}

As shown equation (15), the interaction law $\mathcal{L}$ between normal force $N$ and bending moment $M$ is a primordial relation to establish for super-element 4 . For a beam with a rectangular cross-section, the yield locus is described by the following equation (see [24] for example):

$$
\frac{M}{M_{0}}+\left(\frac{N}{N_{0}}\right)^{2}=1 ; \quad N \leq N_{0}
$$

where $M_{0}$ and $N_{0}$ are respectively the plastic bending moment and the normal plastic force of the cross-section (see Fig. 38). For a T-shaped cross-section, the equation of the yield locus $\mathcal{L}$ is much more complicated (see [18] for more details) and is quite difficult to implement in an analytical procedure. Nevertheless, as suggested by Paik [14], it is sufficient to adopt the parabolic interaction criterion given by formula (36) also for T cross-section. This is the method followed in the present article.

On the other hand, we also know that a fully plastic cross-section submitted to an axial extension $\mathrm{d} \Delta$ and to a rotation $\mathrm{d} \theta$ has to satisfy the normality condition to the yield locus, which states that (see Fig. 38):

$$
\frac{d M}{d N}=-\frac{d \Delta}{d \theta} \Leftrightarrow \frac{2 M_{0} N}{N_{0}^{2}}=\frac{d \Delta}{d \theta} \Leftrightarrow N=\frac{N_{0}^{2}}{2 M_{0}} \cdot \frac{d \Delta}{d \theta}
$$

In order to apply (37), we have to evaluate $\mathrm{d} \Delta / \mathrm{d} \theta=\dot{\Delta} / \dot{\theta}$. These parameters may easily be obtained by some geometrical considerations based on Fig. 23. For the two parts of the impacted beam, we have:

$$
\begin{array}{ll}
\theta_{1}=\frac{\delta \sin \phi}{a_{1}+\delta \cos \phi} & \rightarrow \dot{\theta}_{1}=\frac{a_{1} \sin \phi}{\left(a_{1}+\delta \cos \phi\right)^{2}} \dot{\delta} \\
\theta_{2}=\frac{\delta \sin \phi}{a_{2}-\delta \cos \phi} & \rightarrow \dot{\theta}_{2}=\frac{a_{2} \sin \phi}{\left(a_{2}-\delta \cos \phi\right)^{2}} \dot{\delta} \\
\Delta_{1}=\frac{(\delta \sin \phi)^{2}}{2\left(a_{1}+\delta \cos \phi\right)} & \rightarrow \dot{\Delta}_{1}=\frac{\delta \sin ^{2} \phi}{2} \frac{2 a_{1}+\delta \cos \phi}{\left(a_{1}+\delta \cos \phi\right)^{2}} \dot{\delta} \\
\Delta_{2}=\frac{(\delta \sin \phi)^{2}}{2\left(a_{2}-\delta \cos \phi\right)} & \rightarrow \dot{\Delta}_{2}=\frac{\delta \sin ^{2} \phi}{2} \frac{2 a_{2}-\delta \cos \phi}{\left(a_{2}-\delta \cos \phi\right)^{2}} \dot{\delta}
\end{array}
$$

Consequently, the mathematical expression of $N$ may easily be obtained by substituting $\mathrm{d} \Delta / \mathrm{d} \theta=\dot{\Delta} / \dot{\theta}$ in equation (37) by the corresponding expressions listed in (28). If we do so, we get: 


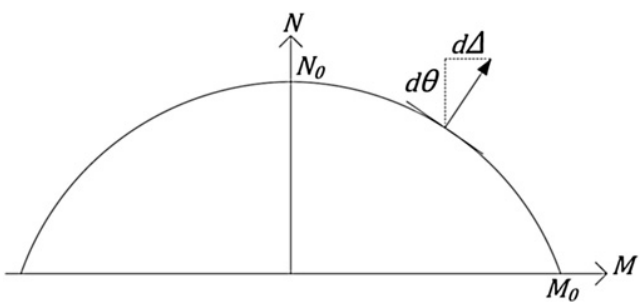

Fig. 38. Parabolic interaction criterion.

$$
N=\frac{N_{0}^{2}}{M_{0}}\left(1-\frac{1}{2} \delta \cos \phi \frac{a_{1}-a_{2}+2 \delta \cos \phi}{a_{1} a_{2}+\delta^{2} \cos ^{2} \phi}\right) ; \quad N \leq N_{0}
$$

Finally, by introducing (36), (38) and (39) in (17), we get the following equation for the crushing force $F$ :

$$
F=2 M_{0} \cdot\left(a_{1} a_{2}+\delta^{2} \cos ^{2} \phi\right) \quad \text { if } N<N_{0}
$$

Of course, this is only valid as long as $N<N_{0}$. When $N=N_{0}$, we have $M=0$ so that (17) may be replaced by:

$$
F \dot{\delta} \sin \phi=N_{0}\left(\dot{\Delta}_{1}+\dot{\Delta}_{2}\right)
$$

In this case, the introduction of (38) in (41) leads to the correction of (40). It has to be replaced by a crushing force given by:

$$
F=N_{0}\left(a_{1}+a_{2}\right) \cdot \delta \sin \phi \cdot \frac{a_{1} a_{2}+\delta^{2} \cos ^{2} \phi-\delta \cos \phi\left(a_{1}-a_{2}\right) / 2}{\left(a_{1}+\delta \cos \phi\right)^{2}\left(a_{2}-\delta \cos \phi\right)^{2}} \quad \text { if } \quad N=N_{0}
$$

\section{Appendix 3.2. Collision scenario}

In order to validate the equations established previously, we have compared them with results given by LS-DYNA. The numerical values defining the collision scenario are listed in Table 8 and the relevant dimensions of the T cross-section are presented on Fig. 39.

\section{Table 8}

Collision parameters for super-element 4 .

\begin{tabular}{lll}
\hline Length of the beam & $a_{1}+a_{2}$ & $8 \mathrm{~m}$ \\
Position of $I_{0}$ & $a_{1}$ & $4 \mathrm{~m}$ \\
& $a_{2}$ & $4 \mathrm{~m}$ \\
Speed of the striking ship & $V_{1}$ & $2 \mathrm{~m} / \mathrm{s}$ \\
Mass of the striking ship & $M_{1}$ & 1426 ton \\
Collision angle & $\phi$ & $45^{\circ}$ \\
Friction coefficient & $\mu$ & 0 \\
\hline
\end{tabular}




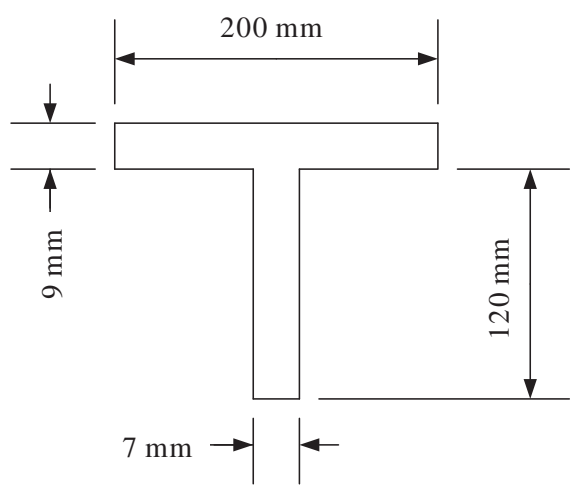

Fig. 39. Cross-section dimensions.

\section{Appendix 4. Details on SE6 development and validation}

We present here some additional mathematical results and also the data needed for numerical validations with LS-DYNA.

\section{Appendix 4.1. Analytical developments}

In this section, we will briefly expose the methodology followed for establishing the crushing resistance of a fold under formation. To do so, we first need to precise the mathematical expression of $\theta$ and $u_{0}$. For example, if we consider the part EID (see Fig. 26) of the fold (the definitions are similar for the second part FID), we have:

$$
\theta=\arccos \left(1-\frac{\delta}{4 H} \sin \phi\right) \quad u_{0}=\frac{1}{2} \frac{\delta^{2} \sin ^{2} \phi}{a_{1}+\delta \cos \phi}
$$

Using these expressions, it is possible to follow the same procedure than the one proposed by Simonsen and Ocakli [21] for a perpendicular collision. We consider in fact that an oblique collision occurring along direction $d_{1}$ on Fig. 26 at point $I_{0}$ and producing a penetration $\delta$ is similar to a right angle collision occurring along direction $d_{2}$ on Fig. 26 at point $I^{\prime}$ and producing a penetration $\delta \sin \phi$. By so doing, we can follow the same method than Simonsen and Ocakli [21], but we have to bear in mind the following differences:

- For the motion of the striking ship, we have to consider the orthogonal projection $\delta \sin \phi$, instead of the total penetration $\delta$.

- Lengths $a_{1}$ and $a_{2}$ don't remain constant during the folding process. A correction need to be apply, so we have to use $a_{1}+\delta \cos \phi$ and $a_{2}-\delta \cos \phi$.

- The two fundamental parameters $\theta$ and $u_{0}$ have to be defined in accordance with formula (43).

If we apply these corrections, we finally obtain the mathematical expression of the crushing force for a given penetration $\delta$ :

$$
F(\delta)=\frac{3}{8 H} \frac{\left(a_{1}+a_{2}\right) \sigma_{0} t_{p}^{2}}{\sqrt{1-\left(1-\frac{\delta}{4 H} \sin \phi\right)^{2}}}+\frac{11}{4} \frac{\left(a_{1}+a_{2}\right) \sigma_{0} t_{p} H}{\left(a_{1}+\delta \cos \phi\right)\left(a_{2}-\delta \cos \phi\right)} \delta \sin ^{2} \phi
$$

Of course, for a perpendicular impact with $\phi=90^{\circ}$, this result reduces to the one obtained by Simonsen en Ocakli [21]. In the previous equation, the parameter $H$ is used to define the total height $4 H$ of one fold, but it remains unknown at this stage. As suggested by Simonsen [21], the analytical expression of $H$ is determined by minimizing the mean crushing force calculated over one fold, i.e.: 


$$
\bar{F}=\frac{1}{4 H} \int_{0}^{4 H} F(\delta) d \delta=\frac{E_{m}+E_{b}}{4 H} \Rightarrow H: \frac{\partial \bar{F}}{\partial H}=0
$$

It can be shown mathematically that this optimal value of $H$ depends on the initial location of the contact point $\left(a_{1}, a_{2}\right)$, the thickness of the plate and the impact angle $\phi$. However, equation (45) is not easy to solve analytically because it leads to quite difficult expression, but this can be achieved by using some numerical procedures, such as the Newton-Raphson method for example.

\section{Appendix 4.2. Collision scenario}

For the numerical validation of super-element, the data defining the collision scenario are those listed in Table 9.

\section{Table 9}

Collision parameters for super-element 6 .

\begin{tabular}{lll}
\hline Total width of the plate & $a_{1}+a_{2}$ & $10 \mathrm{~m}$ \\
Total depth of the plate & $L$ & $12 \mathrm{~m}$ \\
Horizontal initial position of contact point $I_{0}$ & $a_{1}$ & $5 \mathrm{~m}$ \\
& $a_{2}$ & $5 \mathrm{~m}$ \\
Plate thickness & $t_{\mathrm{p}}$ & $2.5 \mathrm{~cm}$ \\
Speed of the striking ship & $V_{1}$ & $2 \mathrm{~m} / \mathrm{s}$ \\
Mass of the striking ship & $M_{1}$ & 1426 ton \\
Tearing strength of steel & $R_{\mathrm{m}}$ & $500 \mathrm{~N} / \mathrm{m}$ \\
\hline
\end{tabular}

\section{References}

[1] Pedersen PT. Review and application of ship collision and grounding analysis procedures. Mar Struct 2010;23:241-62.

[2] Minorsky VU. An analysis of ship collisions with reference to nuclear power plants. J Ship Res 1959;3:1-4.

[3] Wierzbicki T, Culbertson-Driscoll J. Crushing damage of web girders under localized static loads. J Constr Steel Res 1995; 33:199-235.

[4] Wang G, Ohtsubo H. Deformation of ship plate subjected to very large load. In: Proc. of 16th Int. Conf. on Offshore Mech. and Arctic Eng. (OMAE), vol. 2; 1997. p. 173-80.

[5] Simonsen BC. Ship grounding on rock - I. Theory, Mar Struct 1998;10:519-62.

[6] Zhang SM. The mechanics of ship collisions. PhD thesis: Department of Naval Architecture and Offshore Engineering, Technical University of Denmark; 1999.

[7] Hong L, Amdahl J. Crushing resistance of web girders in ship collision and grounding. Mar Struct 2008;21:374-401.

[8] Wang G. Some recent studies on plastic behavior of plates subjected to large impact loads. J Offshore Mech Arctic Eng 2002;124:125-31.

[9] Wang G, Ohtsubo H. Deformation of ship plate subjected to very large load. J Offshore Mech Arctic Eng 1997;119:173-80.

[10] Wang G, Ohtsubo H. An upper-bound solution to the problem of plate tearing. J Mar Sci Technol 1995;1:46-51.

[11] Zhang SM. Plate tearing and bottom damage in ship grounding. Mar Struct 2002;15:101-17.

[12] Wierzbicki T. Concertina tearing of metal plates. Int J Solids Struct 1995;32:2923-43.

[13] Zheng PE. Theorical analysis of wedge cutting through metal plates. PhD thesis: Massachusetts Institute of Technology; 1994.

[14] Paik JK. Ultimate limit state design of steel-plated structures. Chichester: John Wiley and Sons Ltd; 2003.

[17] Amdahl J. Energy absorption in ship-platform impact. PhD thesis: Department of Marine Technology, Norwegian University of Science and Technology; 1983.

[18] Ueda Y, Rashed SMH. The idealized structural unit method and its application to deep girder structures. Comput Struct 1984;18:277-93.

[19] Le Sourne H. A ship collision analysis program based on super-element method coupled with large rotational ship movement analysis tool. ICCGS-2007. In: Int. Conf. on collision and grounding of ships; 2007. p. 131-8.

[20] Le Sourne H, Donner R, Besnier F. External dynamics of ship-submarine collision. ICCGS-2001. In: Int. Conf. on collision and grounding of ships; 2001. p. 137-44.

[21] Simonsen BC, Ocakli H. Experiments and theory on deck girder crushing. Thin-Walled Struct 1999;34:195-216.

[22] LützenM. Ship collision damage. PhD thesis: Department of Mechanical Engineering, Technical University of Denmark; 2001.

[24] Jones N. Structural impact. Cambridge: Cambridge University Press; 1997.

[25] Brown AJ, Sajdak JAW. Modeling longitudinal damage in ship collision. SSC-437; 2005.

[26] Lützen M, Simonsen BC, Pedersen PT. Rapid prediction of damage to struck and striking vessels in a collision even. In: Int. Conf. of ship Struct. for the new millennium: supporting quality in shipbuilding; 2000. Arlington.

[27] Hallquist JO. LS-DYNA theoretical manual. Livermore Software Technology Corporation; 2006. 
[28] Le Sourne H. Collisions bâtiments de surface - rapport n` ${ }^{\circ}$-Etude bibliographique des modèles de rupture utilisés en dynamique rapide - Détermination des seuils de rupture à utiliser dans un calcul de collision. Rapport IRCN n ${ }^{\circ} 01 / \mathrm{RTO}$; 2001.

[29] Törnqvist R. Design of crashworthy ship structures. PhD thesis: Department of Naval Architecture and Offshore Engineering, Technical University of Denmark; 2003.

[30] Ehlers S. Material relation to assess the crashworthiness of ship structures, Department of applied mechanics. Doctoral dissertation: Helsinki University of Technology; 2009.

[31] Brown AJ. Collision scenarios and probabilistic collision damage. J Marine Structures 2002;15(4-5):335-64. 